\title{
Catastrophes and Stable Caustics in Bound States of Hamiltonian Systems
}

John B. Delos

William \& Mary, jbdelos@wm.edu

Follow this and additional works at: https://scholarworks.wm.edu/aspubs

Part of the Physics Commons

\section{Recommended Citation}

Delos, John B., Catastrophes and Stable Caustics in Bound States of Hamiltonian Systems (1987). Journal of Chemical Physics, 86(1), 425-439.

https://doi.org/10.1063/1.452581

This Article is brought to you for free and open access by the Arts and Sciences at W\&M ScholarWorks. It has been accepted for inclusion in Arts \& Sciences Articles by an authorized administrator of W\&M ScholarWorks. For more information, please contact scholarworks@wm.edu. 


\title{
Catastrophes and stable caustics in bound states of Hamiltonian systems
}

\author{
J. B. Delos ${ }^{a)}$ \\ Physics Department, College of William and Mary, Williamsburg, Virginia 23185
}

(Received 21 March 1986; accepted 15 September 1986)

\begin{abstract}
Caustics-envelopes of families of classical trajectories, or boundaries between classically allowed and forbidden regions-correspond to singular points of a phase-space surface called a Lagrangian manifold. According to catastrophe theory, only a limited number of types of caustics are stable under general perturbations of the manifold. Most of the caustics that are found in calculations correspond to members of the canonical list of elementary catastrophes. However, there are some exceptions-examination of trajectories of typical Hamiltonian systems shows that stable structures exist which are not in accord with the stability theorem of catastrophe theory. These exceptional cases are discussed in this paper. They arise because of the special form of the typical Hamiltonian of physical systems.
\end{abstract}

\section{INTRODUCTION}

The relationship between caustics and catastrophes has been discussed in some detail by Berry ${ }^{1}$ and Connor, ${ }^{2}$ who introduced to physicists and chemists some of the mathematical developments that had been made by Thom and by Arnold. ${ }^{3}$ The fundamental result of these studies is the famous classification theorem. While in general, caustics could have practically any geometrical structure, the classification theorem establishes that only a very limited number of structures can be stable or typical. The number of distinct forms of stable or typical caustics is limited by the dimension of configuration space of the system, $N$ (the number of degrees of freedom). For $N$ small, each stable caustic type must correspond to one of Thom's "Elementary Catastrophes." These have been enumerated, and the geometrical structure of each has been analyzed in detail. ${ }^{4}$ In two dimensions, the only stable caustics are supposed to be the so-called "folds" and "cusps," while in three dimensions the list also includes "swallowtails," "elliptic umbilics," and "hyperbolic umbilics."

Many trajectory calculations on a variety of systems have now been carried out. While these calculations generally confirm the utility of the classification theorem, some real and some apparent conflicts with the theory are also found. For example, for bound systems with two degrees of freedom, isolated cusps are rare or nonexistent, and cusps usually occur in pairs, as part of a swallowtail structure. However, according to the classification theorem, the swallowtail is supposed to be stable in three dimensions, but not in two dimensions. Also, in two dimensions, corners appear where two linear caustics meet at a right angle. These corners are characteristic of the hyperbolic umbilic catastrophe (stable for $N=3$ ), and the stable appearance of corners in twodimensional systems is certainly a violation of the classification theorem. Finally, in three dimensions, rectangular corners where three planar caustics meet are very common. These structures are unrelated to any of Thom's elementary catastrophes, so their stable appearance is another violation of the classification theorem.

\footnotetext{
a) Present address: Visiting Fellow, Joint Institute for Laboratory Astro-
} physics, University of Colorado, Boulder, Colorado 80309.
We shall show that this apparent discrepancy between mathematical theory and calculational experience arises because (i) the calculations focus on bound systems, and (ii) the typical Hamiltonian for a physical system is not a completely general function of positions and momenta-instead it is a quadratic form in the momenta,

$$
H=\frac{1}{2} \sum_{i j} T_{i j}(\mathbf{q}) p_{i} p_{j}+V(\mathbf{q}) .
$$

\section{CATASTROPHES AND CAUSTICS}

\section{A. Canonical catastrophes}

Catastrophe theory is a description of singularities of projections of surfaces associated with smooth functions. Suppose we are given a smooth function $\Phi(p, q)$ of $K+N$ real variables: $K$ "internal" variables $\mathrm{p}=\left\{p_{1}, \ldots, p_{K}\right\}$ and $N$ "external" variables $q=\left\{q_{1}, \ldots, q_{N}\right\}$. (For some applications, the variables $p$ are called "state variables" and the variables q are called "control variables"; in the present context, however, the words internal and external seem more

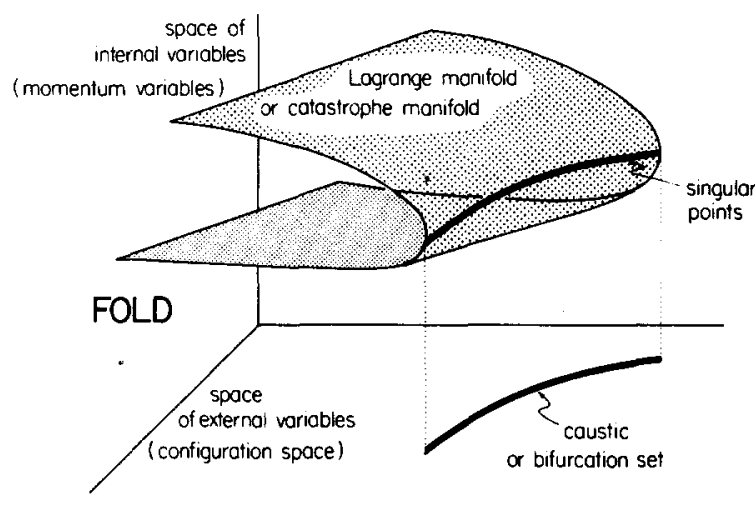

FIG. 1. A Lagrangian manifold is a surface in phase space corresponding to a regular family of trajectories. It is a special case of a catastrophe manifold. In general a catastrophe manifold is a surface in a space of external variables (coordinates, in our case) and internal variables (here, momenta). Singularities of the projection of the catastrophe manifold into the space of external variables (configuration space) are catastrophes. The bifurcation set or caustic is the set of points in configuration space corresponding to the singular points that lie on the manifold. Depicted here is a fold, where the Lagrange manifold turns back over itself. 


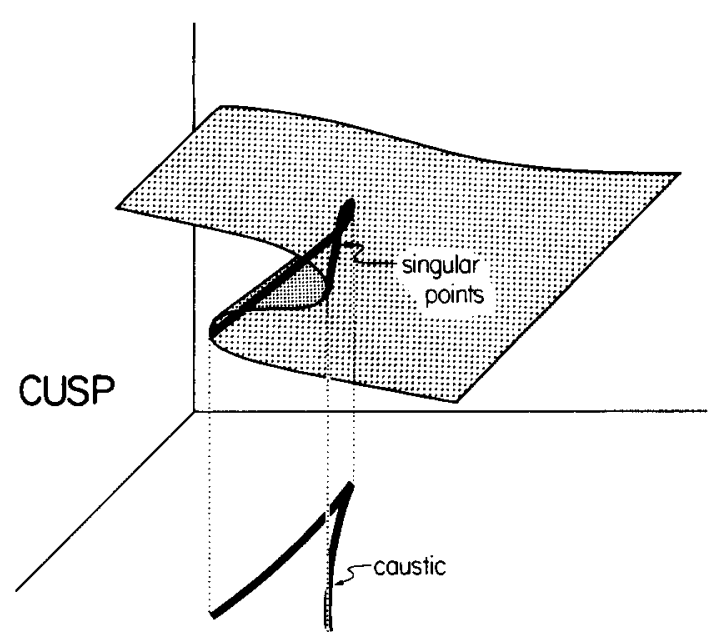

FIG. 2. If configuration space is two dimensional, then only two distinct types of caustics are structurally stable-folds and cusps. A cusp is shown here.

appropriate.) In the $K+N$ dimensional (p,q) space, the locus of points $\left(\mathbf{p}^{0}, \mathbf{q}^{0}\right)$ satisfying the vanishing-gradient condition

$$
\frac{\partial \Phi(\mathbf{p}, \mathbf{q})}{\partial p_{a}}=0 \quad a=1, \ldots, K
$$

forms an $N$-dimensional surface usually called a "catastrophe manifold." We consider the projection of that surface into $q$ space. Singularities of this projection are called catastrophes, and in particular, the points in $q$ space corresponding to singular points on the manifold are called the bifurcation set.

Thom studied the forms such catastrophes could take, and he especially analyzed what happens if the function $\Phi$ is modified by a general smooth perturbation. He considered a catastrophe to be stable or typical if a smooth perturbation to $\Phi$ leaves the structure of the catastrophe essentially unchanged. He established the profound and unexpected result that the number of stable catastrophes is independent of the

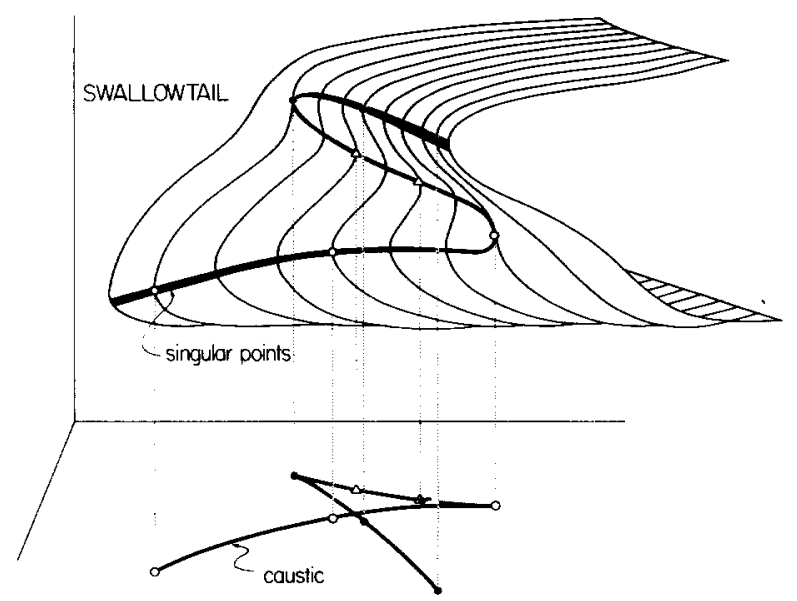

FIG. 3. A dented or twisted fold produces this portion of a swallowtail catastrophe. The complete swallowtail structure emerges when the external space has at least three dimensions. As explained in the text, this portion or slice of the swallowtail is stable in only two dimensions.

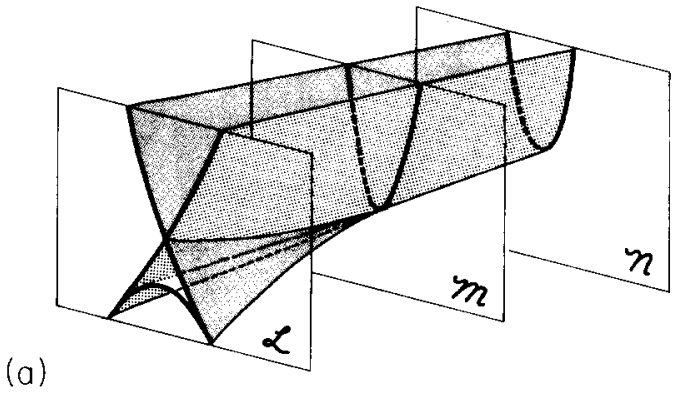

(b)

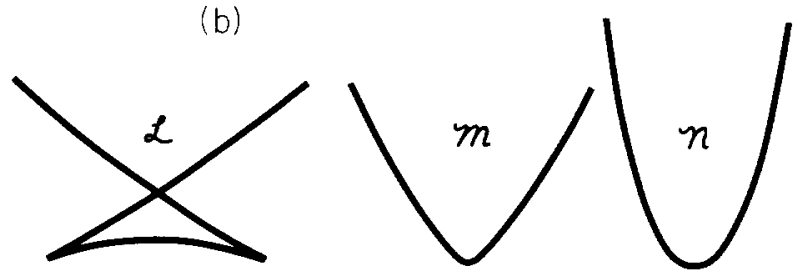

FIG. 4. (a) Bifurcation set or caustic associated with the swallowtail catastrophe; (b) Restriction of the bifurcation set to the planes $\mathscr{L}, \mathscr{H}$, and $\mathscr{N}$ (i.e., intersection of those planes with the bifurcation set). The structures appearing in planes $\mathscr{L}$ and $\mathscr{N}$ are stable in two dimensions, because a small movement of those planes or of the bifurcation set leaves the topological form unchanged. The structure appearing in plane $\mathscr{M}$ is unstable in two dimensions, because a small perturbation changes it to one of the other two structures.

number of "internal" variables $\left\{p_{1}, \ldots, p_{K}\right\}$, and that it depends only on the number of external variables $\left\{q_{1}, \ldots, q_{N}\right\}$. As we mentioned earlier, if there is only one external variable, then the only stable catastrophe is called a fold; if there
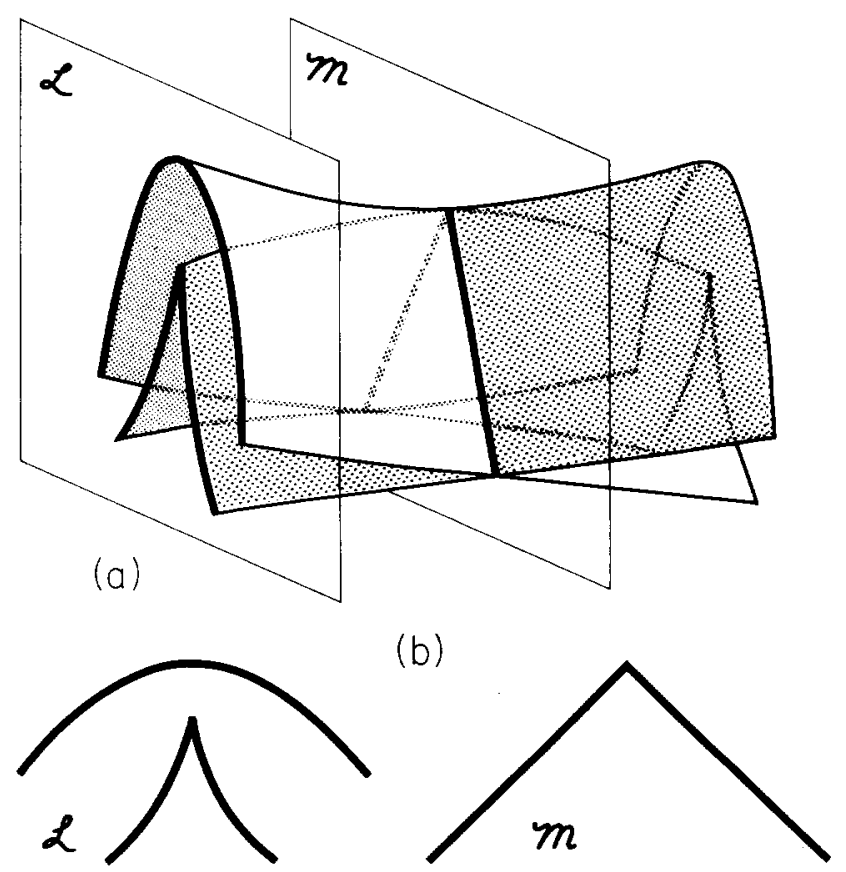

FIG. 5. (a) Bifurcation set (caustic) associated with the hyperbolic umbilic catastrophe. Like the swallowtail, this catastrophe requires an external space of at least three dimensions. (b) Intersection of planes $\mathscr{L}$ and $\mathscr{K}$ with the hyperbolic umbilic bifurcation set. Again the structure appearing in plane $\mathscr{L}$ is stable in two dimensions. According to catastrophe theory the angle or corner seen in plane $\mathscr{M}$ is unstable in two dimensions, because a small perturbation will change it to the fold-and-cusp of plane $\mathscr{L}$. However, calculations on classical dynamical systems show these corners to be stable. This happens because the Hamiltonian is quadratic in the momenta. 

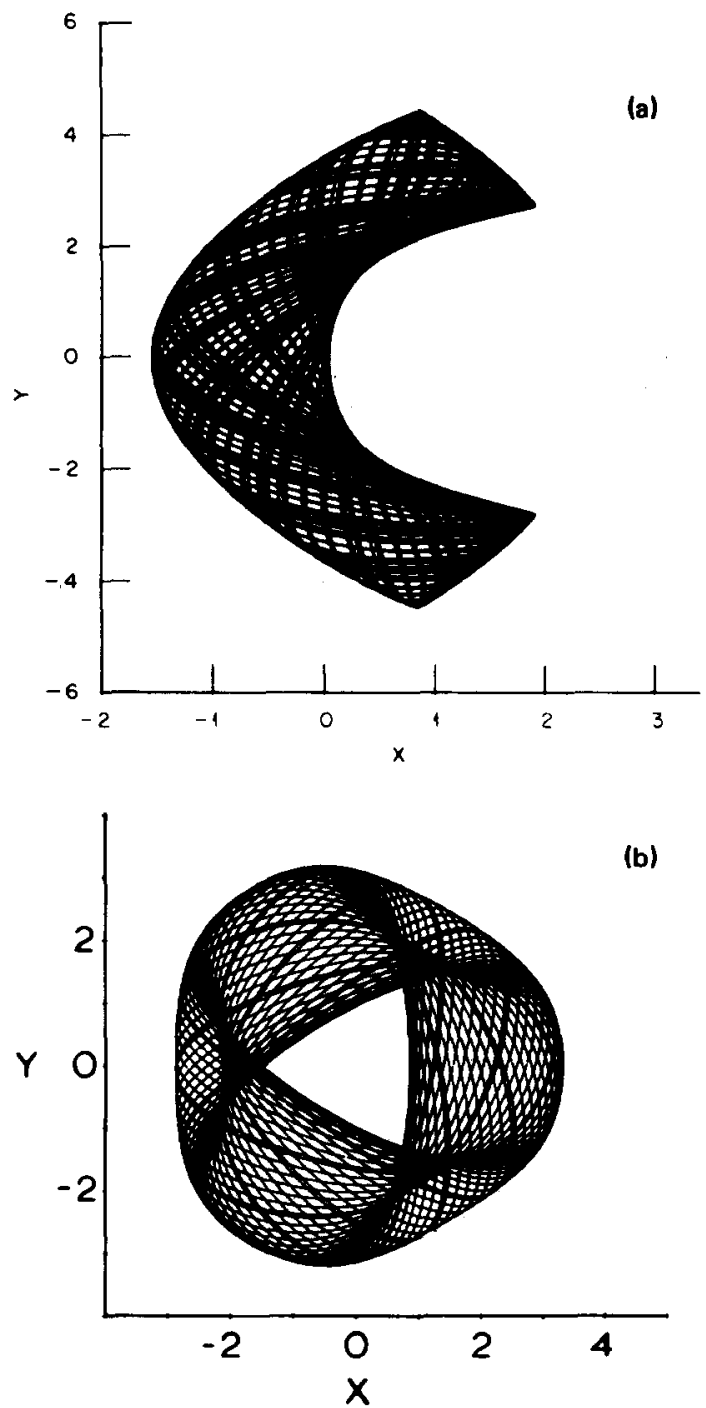
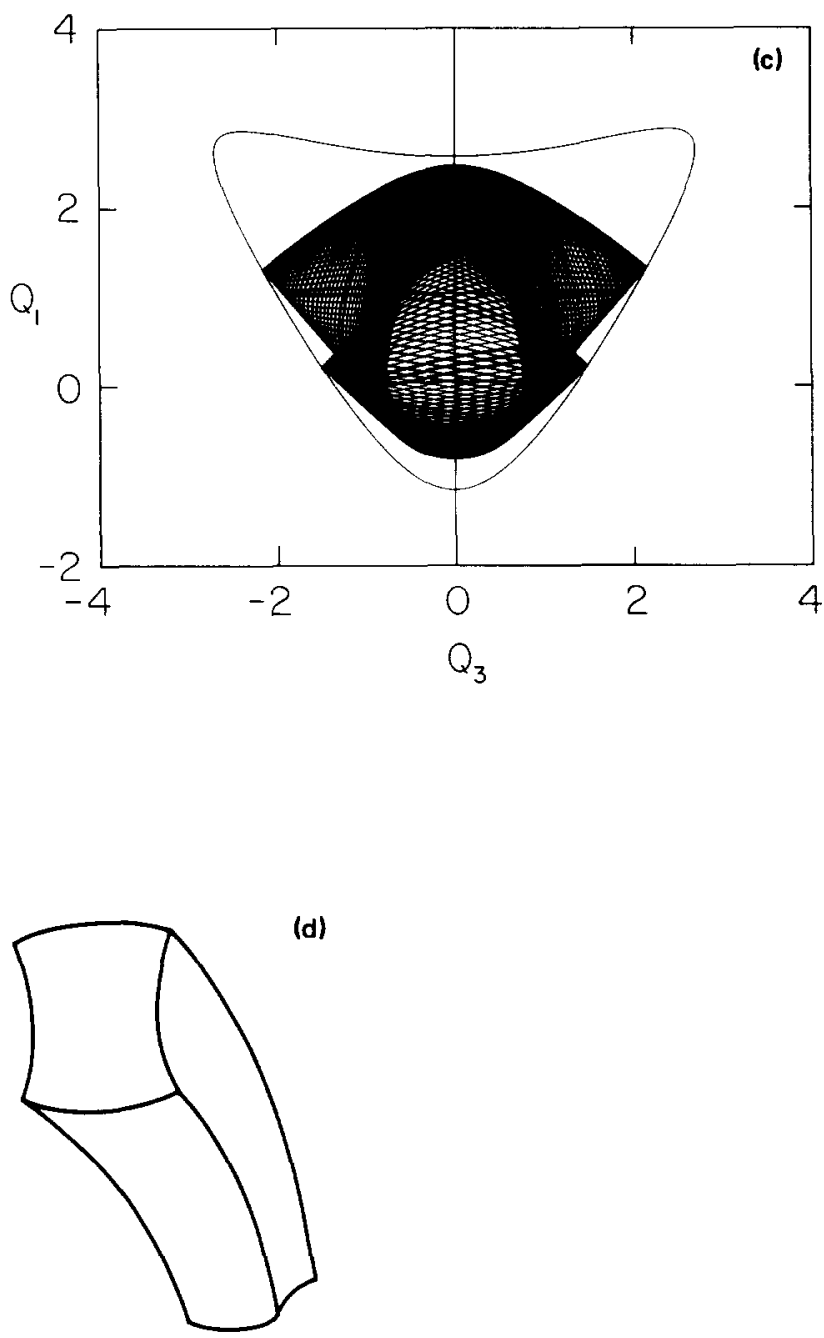

FIG. 6. Typical trajectories for bound Hamiltonian systems with two or three degrees of freedom. (a) Distorted rectangle. The sides correspond to four fold catastrophes, meeting in corners. Note that $X$ and $Y$ axes have different scales. If the scales were the same, the corners would be right angles. Reprinted from Ref. 5(d). (b) Distorted annulus. The outer boundary is a fold caustic while the inner boundary contains three swallowtail structures, each corresponding to the $\mathscr{L}$ slice in Fig. 4. Reprinted from Ref. 5(a); (c) This very distorted rectangle contains folds, corners, and swallowtail sections. Reprinted from Ref. 5(g); (d) The simplest three-dimensional trajectories fill a distorted cube in configuration space, such as the one outlined here. The sides of the cube correspond to folds. The edges and the corners do not correspond to any of the canonical list of catastrophes.

are two, stable catastrophes are limited to folds and cusps. For $N=3$, the list expands to include swallowtails, and elliptic and hyperbolic umbilics, while for $N=4$, besides the lower-order ones, there are butterflies and parabolic umbilics. Some of these are sketched in Figs. 1-5. For more information see Refs. 1-4.

\section{B. Caustics as catastrophes}

A caustic is an envelope of a trajectory or a family of trajectories. Typical examples are shown in Fig. 6. Thom and Arnold ${ }^{3}$ have shown that such envelopes are singularities of projections of catastrophe manifolds, and so they should typically have the forms associated with the canonical catastrophes.

The argument goes as follows. Associated with regular trajectories in configuration space is a surface in phase space known as a Lagrangian manifold. When we examine the interior regions of one of the trajectories in Fig. 6, we see that associated with each configuration space point $\mathbf{q}=\left\{q_{1}, \ldots, q_{N}\right\}$ there is a discrete set of allowable values of the momentum vector $\mathbf{p}=\left\{p_{1}, \ldots, p_{N}\right\}$, and that these allowed values vary smoothly with position $q$. Therefore there is a set of functions $\mathbf{p}(\mathbf{q})$ corresponding to sheets of a surface in phase space, and that surface is the Lagrangian manifold. Arnold $^{3(b)}$ and Maslov and Fedoriuk ${ }^{3(c)}$ have shown that associated with any sufficiently small domain of a Lagrangian manifold, there is a generating function, which can take any of several forms. For systems with $N$ degrees of freedom, a generating function $\widetilde{S}\left(\mathbf{p}_{\alpha}, \mathbf{q}_{\beta}\right)$ is a function of $K$ momenta $\mathbf{p}_{\alpha}=\left\{p_{a} \mid a \in \alpha\right\}$ and $N-K$ coordinates $\mathbf{q}_{\beta}=\left\{q_{b} \mid b \in \beta\right\}$, where the sets $\alpha$ and $\beta$ are disjoint collections of integers between 1 and $N$ containing, respectively, $K$ and $N-K$ elements (this means that the set $\mathbf{p}_{\alpha}, \mathbf{q}_{\beta}$ contains no canonical- 
ly conjugate pairs). The generating function gives the embedding of the domain of the manifold in phase space through the rules

$$
\begin{aligned}
& p_{b}\left(\mathbf{p}_{\alpha}, \mathbf{q}_{\beta}\right)=\frac{\partial \widetilde{S}\left(\mathbf{p}_{\alpha}, \mathbf{q}_{\beta}\right)}{\partial q_{b}} \quad b \in \beta, \\
& q_{a}\left(\mathbf{p}_{\alpha}, \mathbf{q}_{\beta}\right)=\frac{-\partial \widetilde{S}\left(\mathbf{p}_{\alpha}, \mathbf{q}_{\beta}\right)}{\partial p_{a}} \quad a \in \alpha .
\end{aligned}
$$

Let us consider

$$
\Phi\left(\mathbf{p}_{\alpha}, \mathbf{q}_{\alpha}, \mathbf{q}_{\beta}\right)=\widetilde{S}\left(\mathbf{p}_{\alpha}, \mathbf{q}_{\beta}\right)+\sum_{a \in \alpha} p_{a} q_{a} .
$$

The set of $K$ equations

$$
\frac{\partial \Phi}{\partial p_{a}}=0 \quad a \in \alpha
$$

defines the restriction of the manifold to the $\mathbf{p}_{\alpha} \mathbf{q}_{\alpha} \mathbf{q}_{\beta}$ space. Now this is a vanishing-gradient condition of the same form as Eq. (2). Comparing these, we see that the momenta correspond to internal (state) variables and the coordinates correspond to external (control) variables. It follows that a Lagrangian manifold is a special case of a catastrophe manifold. Therefore, the only singularities that are stable under general perturbations of the manifold must be the canonical catastrophes. Since the singular points of the projection of the Lagrange manifold into configuration space are the caustics, it follows that the number of stable caustic structures is limited by the dimension of configuration space. For example if $N=2$, stable caustics should correspond to folds or cusps.

\section{Typical caustics}

Let us now examine Figs. 6 and 7 in more detail. The trajectories and the caustic types shown therein are characteristic of a great variety of physical systems. One finds that folds are very common: all of the smooth parts of the envelopes of the two-dimensional trajectories are fold caustics. Cusps appear occasionally, but rarely in isolation-they usually appear in pairs, as part of a swallowtail structure. ${ }^{6}$ Several examples can be seen in Figs. 6(b), 6(c), and 7(d). This raises the question: Why is the swallowtail, which is said to be a stable structure only for $n \geqslant 3$, nevertheless an important structure for $n=2$ ?

In the figures one also sees corners where two folds meet, almost always at right angles. We will establish that these corners are stable under relevant perturbations of the manifold. However, the simplest catastrophe that contains a corner is the hyperbolic umbilic, in which the corner appears as the most atypical (and therefore supposedly unstable) two-dimensional slice of the bifurcation set. Catastrophe theory is telling us that corners in two dimensions ought to be unstable. How do we resolve this?

Figure 6(d) shows the outline of a three-dimensional trajectory. It possesses sides which correspond to folds, but it also possesses edges where two sides meet, and corners where three edges meet. None of Thom's canonical catastrophes contain such structures, and therefore they are not supposed to be stable.

Why is catastrophe theory misleading us in this way?
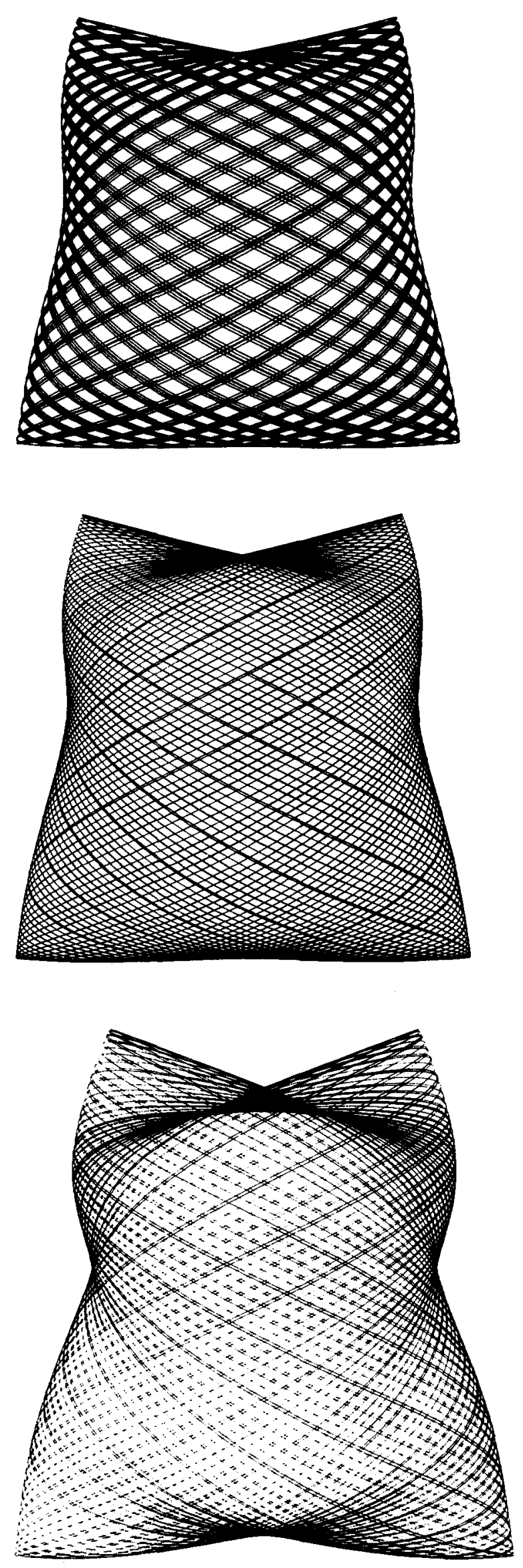

FIG. 7. Development of the swallowtail structure. As the energy changes, the upper caustic is distorted through the sequence of patterns that appears in Fig. 4(b), from $\mathscr{N}$ through $\mathscr{M}$ to $\mathscr{L}$. 
We shall show that the swallowtails appear for a trivial reason, but the corners appear for an interesting reason.

\section{Swallowtails}

In Fig. 6, we have focused attention on systems in which the trajectories occupy a bounded region of configuration space. A fold caustic separates allowed regions from forbidden regions, but a cusp caustic does not (it separates singly allowed from triply allowed regions). Therefore, when a cusp appears in a bounded system, it cannot occur in complete isolation. Hence it can alternately be regarded as one part of some larger catastrophe. The simplest catastrophe that contains a cusp together with a boundary between allowed and forbidden regions is a swallowtail, and in fact when we look at trajectories we most commonly see cusps appearing in pairs, where they represent slices of the swallowtail bifurcation set.

There is no conflict with catastrophe theory here. In fact, this case provides a nice confirmation of the relevance of the classification theorem to the study of caustic structures. Such slices of the swallowtail bifurcation set are stable in two dimensions, in the sense that a general small perturbation to the manifold leaves the topological structure of such a slice unchanged. In Fig. 4(a), we could imagine disturbing the plane $\mathscr{L}$-such a disturbance would not change the essential structure of its intersection with the bifurcation set.

On the other hand, the slice of the bifurcation set obtained from plane $\mathscr{M}$ is topologically unstable, and a small perturbation to the plane or to the manifold changes the structure of the caustic from a simple fold to the three-foldtwo-cusp structure. This also is completely consistent with trajectory calculations. In Figs. 7(a)-7(c) a family of trajectories is shown for a certain physical system at various energies. The sequence of pictures illustrates the continuous "unfolding" or "development" of the swallowtail structure as the energy is varied. Figure 7(b) is the point at which the topological structure changes, and the form of the caustic here is very unstable-a small change of energy produces an important change in the structure. This is just what catastrophe theory tells us.

\section{E. Corners}

Corners appear for a different and much more interesting reason. We shall show that structures which are "generic" among smooth Lagrangian manifolds are not necessarily typical in physics. First let us establish that the corners are stable structures in mechanics.

In the two-dimensional trajectories in Figs. 6 and 7, each corner appears at some point on the equipotential curve having $V(q)=E$; there the momentum is equal to zero. (1) If we fix the Hamiltonian function, fix the energy, and vary the second initial condition specifying the orbit, then the corner persists; it moves to a different point on the equipotential curve, but it maintains its topological structure. In particular, the lines of the corner still meet at right angles. (2) If we vary the energy, the corner still persists. Of course, it moves to a different equipotential curve. ( 3 ) If we change the Hamiltonian by smoothly varying the parameters in the potential energy, the corner again persists, generally shifting its position smoothly. (4) If we examine trajectories of several different Hamiltonians of the form (1), we keep finding corners. Not all trajectories possess corners, but if we start a regular trajectory from rest at a point $\mathbf{q}^{0}$, then a corner appears at $q^{0}$. We therefore cannot avoid thinking of the corners as stable structures. ${ }^{7}$

On the other hand, catastrophe theory, which is absolutely rigorous, tells us that within the class of caustics for $n=2$, corners really are unstable. A general small perturbation to the Lagrangian manifold will change a corner into a different structure, as indicated in Fig. 5. How do we resolve this paradox?

Poston and Stewart ${ }^{4}$ mention the possibility that new or different structures (other than the canonical catastrophes) may appear under certain conditions.

"There are many different notions of structural stability" 4 (b) ..."[T] he notion of stability depends upon the perturbations that we allow... ." 4(c)

Furthermore

" 'Typicality' statements do not mean that for something else to happen is impossible or unphysical. They do mean that something else happening requires special explanation and justification ...."

In particular, noncanonical catastrophes can occur stably if the system possesses some special symmetry, and if they are found,

"[I] $t$ is the job of the mathematical scientist to account for the symmetry which stabilizes them." 4(d)

With the above discussion in mind, let us define or create a distinction between generic and typical Hamiltonian mechanical systems. Let us say that a generic or general Hamiltonian system is described by an arbitrary differentiable function of $p$ 's and $q$ 's, $H(p, q)$. However, let us say that a typical Hamiltonian system is described by a Hamiltonian of the form (1)-i.e., it is quadratic in the momenta (no linear terms).

We consider a certain restricted class of Lagrangian manifolds. (i) The manifold must possess a point which projects to the origin in momentum space $p=0$. (ii) The manifold must possess a small domain containing that point, which domain must admit a diffeomorphic projection into momentum space. Then the domain admits a generator $\widetilde{S}(p)$ such that $q_{i}(\mathbf{p})=\partial \widetilde{S}(\mathbf{p}) / \partial \mathbf{p}_{i}$. (iii) This generator must satisfy a Hamilton-Jacobi equation with a typical Hamiltonian (1).

In the following section, it will be proved that every member of this class of Lagrangian manifolds possesses caustics which form a corner. Therefore, within this restricted class of Lagrangian manifolds, which correspond to a very large subset of physically interesting mechanical systems, the corner is a stable structure.

\section{ANALYSIS OF THE CORNER}

We consider trajectories associated with Hamiltonians of the form (1): the critical assumption is that the expansion of the Hamiltonian in powers of the momenta contains no linear terms in momenta-besides terms independent of momenta, there are only terms that are at least quadratic in momenta (higher terms are allowed by the analysis, but are 
not very relevant to nonrelativistic physics). Let us examine domains of Lagrangian manifolds that have a smooth and smoothly invertible projection into $\mathrm{p}$ space, and which project onto a domain surrounding the origin $\mathbf{p}=0$. The domain of the manifold is characterized by a generating function $\widetilde{S}(\mathbf{p})$ such that $q_{i}(\mathbf{p})=-\partial \widetilde{S}(\mathbf{p}) / \partial p_{i}$. We shall show that Hamiltonians of the form (1) force the generator to take a form such that a corner appears in configuration space at the point $\mathbf{q}^{0}=\mathbf{q}(\mathbf{p}=0)$. Furthermore, we shall show that in suitably defined "normal" coordinates, the lines of the corner meet at right angles. Proofs of these statements are long, and the reader who is not interested in the details might skip to Sec. III F below.

\section{A. Transformation to "normal" coordinates}

The normal variables $(\mathbf{P}, \mathbf{Q})$ are defined in the following way. The origin of $\mathbf{Q}$ coordinates, $\mathbf{Q}=0$, is taken to be the point $\mathbf{q}^{0}$ corresponding to $\mathbf{p}=0$; i.e., the manifold point which projects onto the point $\mathbf{P}=0$ in momentum space also projects onto the point $\mathbf{Q}=0$ in configuration space. (The origin of coordinates is at the corner that will emerge from the analysis.) Furthermore, the $Q$ 's are taken to be linear combinations of $q$ 's such that the transformed kinetic energy matrix evaluated at $\mathbf{q}^{0}$ is equal to the unit matrix. Such coordinates can be constructed by a modification of the method that is used to calculate normal modes of vibration (but of course the $Q$ 's are not the same normal coordinates that would be obtained if the expansion had been carried out about the equilibrium point). The transformed Hamiltonian is

$$
\begin{aligned}
& \widehat{H}(\mathbf{P}, \mathbf{Q})=\frac{1}{2} \sum_{i j} \widehat{T}_{i j}(\mathbf{Q}) P_{i} P_{j}+\widehat{V}(\mathbf{Q}), \\
& \widehat{T}(\mathbf{0})=1, \\
& \widehat{V}(\mathbf{0})=E .
\end{aligned}
$$

A derivation is given in the Appendix.

This Hamiltonian is now expanded in powers of $Q$, and the leading terms are

$$
\begin{aligned}
& \hat{H}-E=\sum_{i}\left(\frac{1}{2} P_{i}^{2}-F_{i} Q_{i}\right)+\cdots, \\
& F_{i}=-\frac{\partial \widehat{V}}{\partial Q_{i}} .
\end{aligned}
$$

Higher terms in Eq. (7) contain either quadratic products $Q_{i} Q_{j}$ or cubic products $Q_{i} P_{j} P_{k}$ or higher degree products. The $Q$ 's are referred to as normal variables because of the simple form of Eq. (7). [Perhaps we should emphasize here that we are not assuming that higher-order terms in $H$ have no effect on the trajectory as a whole. It is rather that the higher-order terms are irrelevant. Our analysis, like catastrophe theory, deals only with local behavior of caustics, and this local behavior is determined entirely by the leading terms in Eq. (7).]

The generator $\widetilde{S}(p)$ can be reexpressed as a new function of the new momenta, $\widetilde{S}(\mathbf{p})=\widehat{S}(\mathbf{P})$, and the resulting function must satisfy the Hamilton-Jacobi equation,

$$
\hat{H}\left(\mathbf{P},-\frac{\partial \widehat{S}}{\partial \mathbf{P}}\right)-E=0
$$

for which the lowest-order terms are

$$
\sum_{i}\left(\frac{1}{2} P_{i}^{2}+F_{i} \frac{\partial \hat{S}}{\partial P_{i}}\right)+\cdots=0 .
$$

Now let us expand $\hat{S}(\mathbf{P})$ in a Taylor series in $\mathbf{P}$,

$$
\begin{aligned}
\widehat{S}(\mathbf{P})= & S_{0}+\sum_{i} S_{i} P_{i} \\
& +\frac{1}{2} \sum_{i j} S_{i j} P_{i} P_{j}+\frac{1}{6} \sum_{i j k} S_{i j k} P_{i} P_{j} P_{k}+\cdots .
\end{aligned}
$$

The constant is irrelevant. The linear terms vanish because of the definition of the origin of $Q$ coordinates

$$
Q_{i}(\mathbf{P}=0)=-\left.\frac{\partial \hat{S}(\mathbf{P})}{\partial P_{i}}\right|_{P=0}=-S_{i}=0 .
$$

The quadratic terms vanish because when $\hat{S}(P)$ is put into Eq. (9), the quadratic terms produce terms which are linear in $P$ 's, and which cannot be canceled by anything else in that equation. [The kinetic energy is quadratic in $P$ 's, and any product $\left(\partial \widehat{S} / \partial P_{i}\right)\left(\partial \widehat{S} / \partial P_{j}\right)$ arising from higher-order terms in Eq. (9) is also at least quadratic in $P$ 's.] Therefore the lowest terms in $\widehat{S}(\mathbf{P})$ are cubic.

\section{B. Analytical geometry of the corner}

The analysis up to this point applies to systems with any number of degrees of freedom. Let us now restrict ourselves to the case $N=2$.

The general cubic- and-higher generator is in this case

$\widehat{S}(\mathbf{P})=\frac{1}{3} A P_{1}^{3}+\frac{1}{2} B P_{1}^{2} P_{2}+\frac{1}{2} C P_{1} P_{2}^{2}+\frac{1}{3} D P_{2}^{3}+\cdots$,

where $A, B, C, D$ are constants. The equations for the embedding of the manifold in phase space are

$$
\begin{aligned}
& -Q_{1}\left(P_{1} P_{2}\right)=\frac{\partial \hat{S}}{\partial P_{1}}=A P_{1}^{2}+B P_{1} P_{2}+\frac{1}{2} C P_{2}^{2}+\cdots, \\
& -Q_{2}\left(P_{1} P_{2}\right)=\frac{\partial \hat{S}}{\partial P_{2}}=\frac{1}{2} B P_{1}^{2}+C P_{1} P_{2}+D P_{2}^{2}+\cdots
\end{aligned}
$$

Applying the Hamilton-Jacobi equation (8) to the generator (12), and collecting in powers of $P$ 's using the fact that the lowest-degree parts of $H$ have the form (7a), we obtain

$$
\begin{aligned}
& \frac{1}{2}\left(P_{1}^{2}+P_{2}^{2}\right)+\left(A P_{1}^{2}+B P_{1} P_{2}+\frac{1}{2} C P_{2}^{2}\right) F_{1} \\
& \quad+\left(\frac{1}{2} B P_{1}^{2}+C P_{1} P_{2}+D P_{2}^{2}\right) F_{2}+\cdots=0 .
\end{aligned}
$$

Since $P$ 's are independent variables, this implies a set of simultaneous linear equations for the constants $A-D$,

$$
\begin{aligned}
& F_{1} A+\frac{1}{2} F_{2} B=-\frac{1}{2}, \\
& F_{1} B+F_{2} C=0, \\
& \frac{1}{2} F_{1} \mathrm{C}+F_{2} D=-\frac{1}{2} .
\end{aligned}
$$

Since $F$ 's are determined from the Hamiltonian, these are three equations in four unknowns. They therefore admit a one-parameter family of solutions-one of the coefficients (we will choose $B$ ) is arbitrary, and the others are deter- 
mined from it:

$$
\begin{aligned}
& A=-\left(\frac{1}{2}+\frac{1}{2} F_{2} B\right) / F_{1}, \\
& C=-F_{1} B / F_{2}, \\
& D=-\left(\frac{1}{2}-\frac{1}{2} F_{1}^{2} B / F_{2}\right) / F_{2} .
\end{aligned}
$$

The qualitative properties of the manifold near $\mathbf{P}=0$ are determined by the cubic terms in $\hat{S}$, or the quadratic terms in $\mathbf{Q}(\mathbf{P})$, so henceforth we will ignore all higher terms. $^{8}$

Caustics are singularities of the projection of the manifold into $Q$ space, and they occur when a Jacobian vanishes

$$
\frac{\partial\left(Q_{1}, Q_{2}\right)}{\partial \text { (intrinsic manifold coordinates) }}=0 \text {. }
$$

Whatever we might choose for "intrinsic manifold coordinates," the manifold locally has a good projection into $P$ space, so

$$
\frac{\partial \text { (intrinsic manifold coordinates })}{\partial\left(P_{1}, P_{2}\right)} \neq 0
$$

and therefore the caustics are determined by the condition

$$
\frac{\partial\left[Q_{1}\left(P_{1}, P_{2}\right), Q_{2}\left(P_{1}, P_{2}\right)\right]}{\partial\left(P_{1}, P_{2}\right)}=0 \text {. }
$$

Evaluation of this Jacobian leads to the equation

$$
\left(2 A C-B^{2}\right) P_{1}^{2}+(4 A D-B C) P_{1} P_{2}+\left(2 B D-C^{2}\right) P_{2}^{2}=0
$$

or, defining $\rho=P_{1} / P_{2}$,

$$
\begin{aligned}
& \rho^{2}+\beta \rho+\gamma=0, \\
& \beta=(4 A D-B C) /\left(2 A C-B^{2}\right), \\
& \gamma=\left(2 B D-C^{2}\right) /\left(2 A C-B^{2}\right) .
\end{aligned}
$$

The roots of this quadratic equation (19) may be denoted $\rho_{+}$ and $\rho_{-}$:

$$
\rho_{ \pm}=\frac{1}{2}\left[-\beta \pm\left(\beta^{2}-4 \gamma\right)^{1 / 2}\right] \text {. }
$$

These roots $\rho_{ \pm}$satisfy another polynomial equation that occurs repeatedly in proofs given below. Let us consider again the quadratic equation (19a) whose roots are $\rho_{+}$and $\rho_{-}$, and let us multiply that equation by the factor $\rho_{ \pm}-f$ with $f=F_{1} / F_{2}$ :

$$
\begin{aligned}
& \left(\rho_{ \pm}^{2}+\beta \rho_{ \pm}+\gamma\right)\left(\rho_{ \pm}-f\right)=0, \\
& \rho_{ \pm}^{3}+(\beta-f) \rho_{ \pm}^{2}+(\gamma-\beta f) \rho_{ \pm}-\gamma f=0 .
\end{aligned}
$$

Now using Eq. (19b) together with the evaluations

$$
\begin{aligned}
& 4 A D-B C=\left[F_{2}+\left(F_{2}^{2}-F_{1}^{2}\right) B\right] / F_{1} F_{2}^{2}, \\
& 2 A C-B^{2}=B / F_{2}
\end{aligned}
$$

one can in a few minutes of scribbling show that

$$
\begin{aligned}
& \beta-f=2(C-A) / B, \\
& \gamma-\beta f=2(D-B) / B,
\end{aligned}
$$

and therefore that Eq. (21b) is equivalent to

$$
\frac{1}{2} B \rho_{ \pm}^{3}+(C-A) \rho_{ \pm}^{2}+(D-B) \rho_{ \pm}-\frac{1}{2} C=0 .
$$

This equation will appear again later.

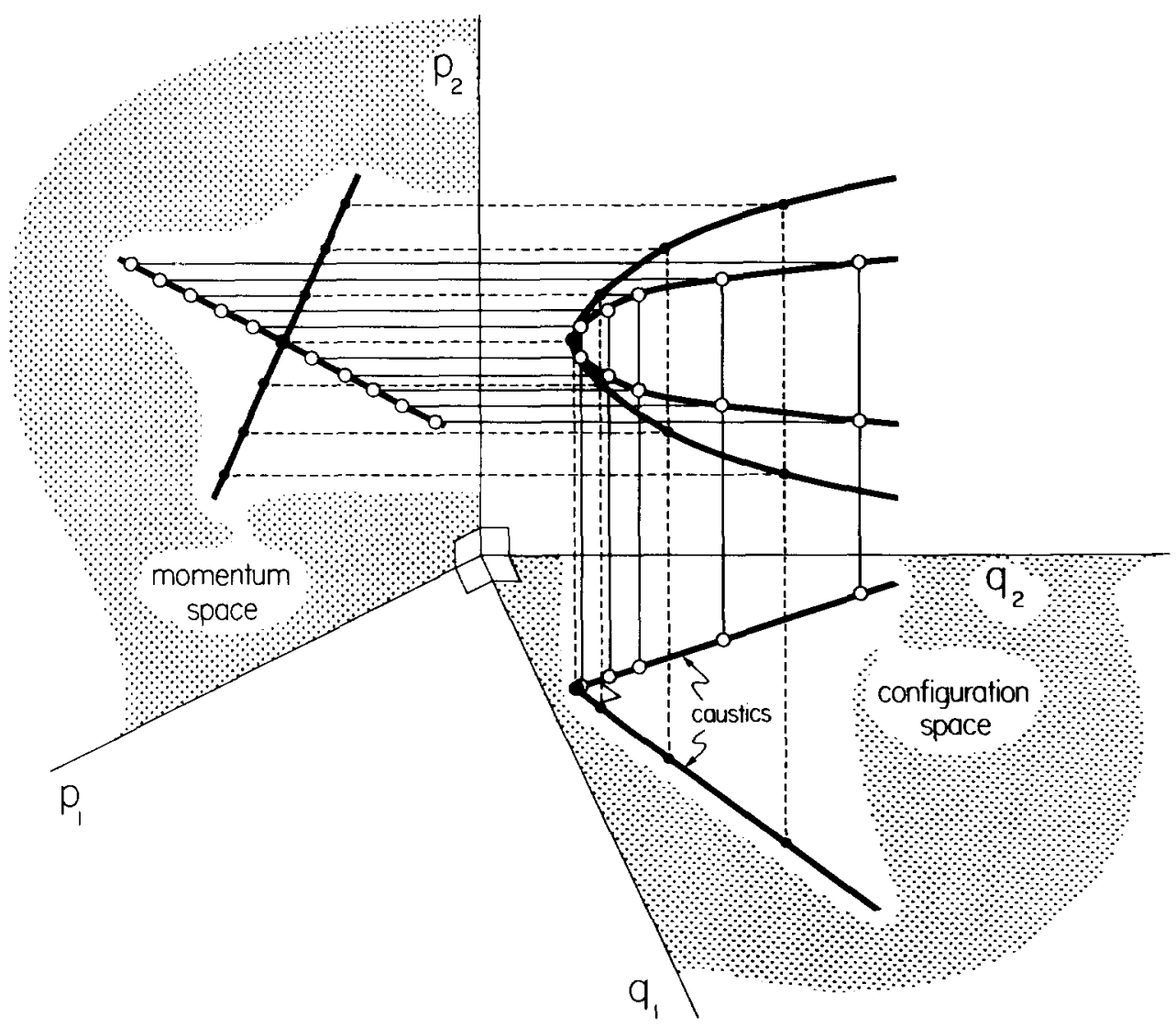

FIG. 8. "Impossible" figure illustrating the corner associated with a hyperbolic umbilic. Phase space has four dimensions, with axes $p_{1} p_{2} q_{1} q_{2}$. The projections of these axes into the plane of the paper are four straight lines meeting at an origin. One must imagine that these lines are all orthogonal (this is most easily done by alternately hiding the $p_{1}, q_{1}$, or $q_{2}$ axes). The $q_{1} q_{2}$ plane is configuration space and the $p_{1} p_{2}$ plane is momentum space. The two parabolic curves lie upon a smooth twodimensional surface in the four-dimensional phase space. They represent the set of singular points on the Lagrangian manifold. The parabola with black dots projects to a straight line in momentum space. Both branches of that parabola project to a single half-line in configuration space. Likewise the two branches of the parabola with hollow dots project to a straight line in momentum space but to a half-line in configuration space. The caustics in configuration space meet at a right angle, as do the singular lines in momentum space. Such a structure is impossible in three dimensions, but it does exist in four-dimensional space. 
The two roots $\rho_{ \pm}$define two straight lines through the origin of the $P_{1} P_{2}$ plane. These two lines are named $\pi_{+}$and $\pi_{-}$, each being defined by an equation of the form $P_{1}=\rho_{ \pm} P_{2}$. These two straight lines are the projections of the singular points of the manifold into the $P_{1} P_{2}$ plane.

These two lines meet at right angles. ${ }^{9}$ To prove this, note that the condition required is

$$
\frac{d P_{1}^{+}\left(P_{2}\right)}{d P_{2}}=-\left[\frac{d P_{1}^{-}\left(P_{2}\right)}{d P_{2}}\right]^{-1}
$$

or

$$
\rho_{+}=-1 / \rho_{-} .
$$

Now the constant term in a quadratic equation of the form (19) is equal to the product of the roots, so let us examine that term:

$$
\begin{aligned}
\rho_{+} \rho_{-} & =\gamma \\
& =\left(2 B D-C^{2}\right) /\left(2 A C-B^{2}\right) \\
& =\frac{-B / F_{2}+B^{2} F_{1}^{2} / F_{2}^{2}-B^{2} F_{1}^{2} / F_{2}^{2}}{B / F_{2}+B^{2}-B^{2}} \\
& =-1
\end{aligned}
$$

and the lines are indeed perpendicular.

These two lines in P space project out via Eqs. (13) to two curves on the manifold, and these curves are the locus of singular points (Fig. 8). The projections of these curves into $\mathbf{Q}$ space are the caustics. Thus the caustics can be given parametrically by the equations

$$
Q_{I}^{ \pm}\left(P_{2}\right)=Q_{I}\left[P_{1}^{ \pm}\left(P_{2}\right), P_{2}\right] \quad I=1,2,
$$

i.e., with $P_{2}$ as the independent variable, the " + " caustic is the line

$$
\begin{aligned}
& Q_{1}^{+}\left(P_{2}\right)=\left(A \rho_{+}^{2}+B \rho_{+}+\frac{1}{2} C\right) P_{2}^{2}, \\
& Q_{2}^{+}\left(P_{2}\right)=\left(\frac{1}{2} B \rho_{+}^{2}+C \rho_{+}+D\right) P_{2}^{2},
\end{aligned}
$$

and the " - " caustic is the line

$$
\begin{aligned}
& Q_{1}^{-}\left(P_{2}\right)=\left(A \rho_{-}^{2}+B \rho_{-}+\frac{1}{2} C\right) P_{2}^{2}, \\
& Q_{2}^{-}\left(P_{2}\right)=\left(\frac{1}{2} B \rho_{-}^{2}+C \rho_{-}+D\right) P_{2}^{2} .
\end{aligned}
$$

These are again two straight lines through the origin, for

$$
\begin{aligned}
\sigma_{ \pm} & =\frac{Q_{1}^{ \pm}\left(P_{2}\right)}{Q_{2}^{ \pm}\left(P_{2}\right)}=\frac{A \rho_{ \pm}^{2}+B \rho_{ \pm}+\frac{1}{2} C}{\frac{1}{2} B \rho_{ \pm}^{2}+C \rho_{ \pm}+D} \\
& =\text { constant }
\end{aligned}
$$

The two caustics therefore form a corner.

Furthermore, in $\mathbf{Q}$ space as well as $\mathbf{P}$ space, the lines meet at right angles. The necessary and sufficient condition for this is that

$$
\sigma_{+} \sigma_{-}=-1
$$

and this follows from the fact that:
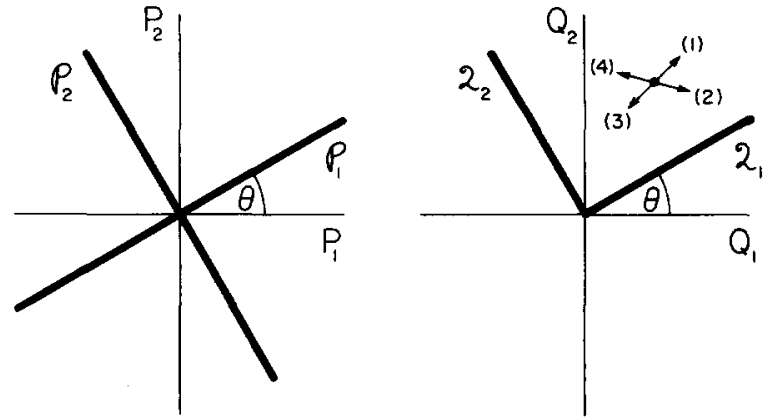

FIG. 9. Canonical rotation in position and momentum space from $P_{1} P_{2} Q_{1} Q_{2}$ to $\mathscr{P}_{1} \mathscr{P}_{2} \mathscr{Q}_{1} \mathscr{Q}_{2}$ coordinates. On the right-hand side is also shown our conventions for labelling branches of the Lagrangian manifold.

$$
\begin{aligned}
& \sigma_{+}=\rho_{+}, \\
& \sigma_{-}=\rho_{-} .
\end{aligned}
$$

These equations are proved by rewriting Eq. (24) in the form

$\frac{1}{2} B \rho_{ \pm}^{3}+C \rho_{ \pm}^{2}+D \rho_{ \pm}=A \rho_{ \pm}^{2}+B \rho_{ \pm}+\frac{1}{2} C$

or

$$
\begin{aligned}
\rho_{ \pm} & =\left(A \rho_{ \pm}^{2}+B \rho_{ \pm}+\frac{1}{2} C\right) /\left(\frac{1}{2} B \rho_{ \pm}^{2}+C \rho_{ \pm}+D\right) \\
& =\sigma_{ \pm} .
\end{aligned}
$$

\section{Natural coordinates for the corner}

The above results suggest that a change of variables might put the generator in a simpler form, and in fact we still have to show that $\widehat{S}$ corresponds to the hyperbolic umbilic catastrophe. We have shown that the projection of the singular points into the $P_{1}, P_{2}$ plane forms two straight lines that meet in a right angle at the origin (Fig. 9). Therefore it is natural to consider a rotation of momentum coordinates such that the new axes are aligned with these projected singular points.

Let us define $\mathscr{P}_{1} \mathscr{P}_{2}$ as rotated $P_{1} P_{2}$ coordinates,

$$
\left[\begin{array}{l}
P_{1} \\
P_{2}
\end{array}\right]=\left[\begin{array}{cc}
\cos \theta & -\sin \theta \\
\sin \theta & \cos \theta
\end{array}\right]\left[\begin{array}{l}
\mathscr{P}_{1} \\
\mathscr{P}_{2}
\end{array}\right],
$$

i.e., given a point in the $\mathbf{P}$ plane represented by components $P_{1} P_{2}$, then the same point is represented in rotated coordinates by the components $\mathscr{P}_{1} \mathscr{P}_{2}$. By choosing

$$
\tan \theta=1 / \rho_{-}
$$

we make the $\mathscr{P}_{1}$ axis correspond to the singular line $\pi_{-}$. By convention, the angle $\theta$ lies between $-\pi / 2$ and $\pi / 2$.

Let us now reexpress $\hat{S}$ in terms of these rotated coordinates. Combining Eqs. (33) with (12), and abbreviating $c=\cos \theta, s=\sin \theta$, a straightforward calculation gives

$$
\begin{aligned}
\mathscr{S}(\mathscr{P}) \equiv \hat{S}[P(\mathscr{P})]= & \frac{1}{} A\left(c^{3} \mathscr{P}_{1}^{3}-3 c^{2} s \mathscr{P}_{1}^{2} \mathscr{P}_{2}+3 c s^{2} \mathscr{P}_{1} \mathscr{P}_{2}^{2}-s^{3} \mathscr{P}_{2}^{3}\right) \\
& +\frac{1}{2} B\left[c^{2} \mathscr{P}_{1}^{3}+\left(c^{3}-2 c s^{2}\right) \mathscr{P}_{1}^{2} \mathscr{P}_{2}+\left(s^{3}-2 c^{2} s\right) \mathscr{P}_{1} \mathscr{P P}_{2}^{2}+c s^{2} \mathscr{P P}_{2}^{3}\right] \\
& +\frac{1}{2} C\left[c s^{2} \mathscr{P}_{1}^{3}-\left(s^{3}-2 c^{2} s\right) \mathscr{P}_{1}^{2} \mathscr{P}_{2}+\left(c^{3}-2 c s^{2}\right) \mathscr{P}_{1} \mathscr{P}_{2}^{2}-c^{2} s \mathscr{P}_{2}^{3}\right] \\
& +\frac{1}{3} D\left[s^{3} \mathscr{P}_{1}^{3}+3 c s^{2} \mathscr{P}_{1}^{2} \mathscr{P}_{2}+3 c^{2} s_{P_{1}} \mathscr{P}_{2}^{2}+c^{3} \mathscr{P}_{2}^{3}\right] .
\end{aligned}
$$



$B^{\prime}$ :

We need to examine the coefficient of $\mathscr{P}_{1}^{2} \mathscr{P}_{2} \longrightarrow$ call it

$$
\begin{aligned}
B^{\prime}= & -\frac{1}{3} A\left(3 c^{2} s\right)+\frac{1}{2} B\left(c^{3}-2 c s^{2}\right) \\
& -\frac{1}{2} C\left(s^{3}-2 c^{2} s\right)+\frac{1}{3} D\left(3 c s^{2}\right) .
\end{aligned}
$$

This can be evaluated using

$$
\begin{aligned}
& \sin \theta=\tan \theta /\left(1+\tan ^{2} \theta\right)^{1 / 2}, \\
& \cos \theta=\left(1+\tan ^{2} \theta\right)^{-1 / 2} .
\end{aligned}
$$

(The positive square root is involved in both of these expressions; this follows from the above-noted convention that $-\pi / 2<\theta<\pi / 2$.) Substituting Eq. (37) into Eq. (36), multiplying the expression by $(1+\tan \theta)^{3 / 2}$, and then using $\mathrm{Eq}$. (34), we obtain

$$
\begin{aligned}
B^{\prime}= & -A / \rho_{-}+\frac{1}{2} B\left(1-2 / \rho_{-}^{2}\right) \\
& -\frac{1}{2} C\left(1-2 \rho_{-}^{2}\right) / \rho_{-}^{3}+D / \rho_{-}^{2}
\end{aligned}
$$

and multiplying by $\rho_{-}^{3}$ and rearranging terms we find that the coefficient is

$\rho_{-}^{3} B^{\prime}=\frac{1}{2} B \rho_{-}^{3}+(C-A) \rho_{-}^{2}+(D-B) \rho_{-}-\frac{1}{2} C$

which, according to $\mathrm{Eq}$. (24), is exactly equal to zero.

If we apply the same process to the coefficient of $\mathscr{P}_{1} \mathscr{P}_{2}^{2}$ in Eq. (35), and if for $\tan \theta$ we substitute $-\rho_{+}$(which is equal to $1 / \rho_{-}$), then the magical equation (24) again appears, so this coefficient also vanishes. It follows that in these rotated coordinates, $\mathscr{S}$ is given simply by

$$
\mathscr{S}=\frac{1}{3} A^{\prime} \mathscr{P}_{1}^{3}+\frac{1}{3} D^{\prime} \mathscr{P}_{2}^{3}
$$

with

$$
\begin{aligned}
& A^{\prime}=\frac{1}{3} A c^{3}+\frac{1}{2} B c^{2} s+\frac{1}{2} C c s^{2}+\frac{1}{3} D s^{3}, \\
& D^{\prime}=-\frac{1}{3} A s^{3}+\frac{1}{2} B c s^{2}-\frac{1}{2} C c^{2} s+\frac{1}{3} D c^{3} .
\end{aligned}
$$

The geometry of the singular points can be calculated very quickly from this form. Corresponding to the rotation of momenta (33), there is a rotation of coordinates (Fig. 9)

$$
\left[\begin{array}{l}
Q_{1} \\
Q_{2}
\end{array}\right]=\left[\begin{array}{cc}
\cos \theta & -\sin \theta \\
\sin \theta & \cos \theta
\end{array}\right]\left[\begin{array}{l}
\mathscr{Q}_{1} \\
\mathscr{Q}_{2}
\end{array}\right]
$$

With such rotations, the full transformation $P_{1} P_{2} Q_{1} Q_{2}$ $\rightarrow \mathscr{P}_{1} \mathscr{P}_{2} \mathscr{Q}_{1} \mathscr{Q}_{2}$ is canonical. Therefore the Lagrangian manifold is given in these coordinates by

$$
\begin{aligned}
& \mathscr{Q}_{1}\left(\mathscr{P}_{1}, \mathscr{P}_{2}\right)=-\frac{\partial \mathscr{P}}{\partial \mathscr{P}_{1}}=-A^{\prime} \mathscr{P}_{1}^{2}, \\
& \mathscr{Q}_{2}\left(\mathscr{P}_{1}, \mathscr{P}_{2}\right)=-\frac{\partial \mathscr{P}}{\partial \mathscr{P}_{2}}=-D^{\prime} \mathscr{P}_{2}^{2} .
\end{aligned}
$$

The Jacobian defining the singular points is just

$$
\frac{\partial\left(\mathscr{Q}_{1}, \mathscr{Q}_{2}\right)}{\partial\left(\mathscr{P}_{1}, \mathscr{P}_{2}\right)}=4 A^{\prime} D^{\prime} \mathscr{P}_{1} \mathscr{P}_{2}
$$

which vanishes on the lines $\mathscr{P}_{1}=0$ and $\mathscr{P}_{2}=0$. Therefore the caustics are the half-lines $\mathscr{Q}_{1}=0$ with $\operatorname{sgn}\left(\mathscr{Q}_{2}\right)$ fixed, and $\mathscr{Q}_{2}=0$ with sgn $\left(\mathscr{Q}_{1}\right)$ fixed.

\section{Stability of the corner}

The expression

$$
\frac{1}{3} \mathscr{P}_{1}^{\prime 3}+\frac{1}{3} \mathscr{P}_{2}^{\prime 3}
$$

is a standard form for the "germ" of the hyperbolic umbilic catastrophe, and the expression

$$
\frac{1}{3} \mathscr{P}_{1}^{\prime 3}+\frac{1}{3} \mathscr{P}_{2}^{\prime 3}-a_{1} \mathscr{P}_{1}^{\prime}-a_{2} \mathscr{P}_{2}^{\prime}-a_{3} \mathscr{P}_{1}^{\prime} \mathscr{P}_{2}^{\prime}
$$

represents a "universal unfolding" of this catastrophe. The terms germ and unfolding are defined in various references on catastrophe theory. ${ }^{1,2,4}$ Their essential meaning is the following. Given a manifold with a generator of the form (44), we may consider general smooth perturbations of the generator, which produce smooth perturbations of the manifold. It can be shown that small perturbations of the generator (44) lead to essential changes in the geometrical structure of the caustics. However, given any manifold which was obtained by an arbitrary small perturbation of generator (44), there exists a smooth change of coordinates such that the generator of the perturbed manifold has exactly the form (45), with $a_{1}, a_{2}$, and $a_{3}$ constants. Therefore, all possible caustic structures associated with any perturbation of Eq. (44) can be found by examining the structures associated with the universal unfolding (45), for various values of the parameters $a_{1}, a_{2}$, and $a_{3}$.

Now the generator (39) can be transformed to the form (44) by appropriate rescaling of the $\mathscr{P}$ 's $\left(\mathscr{P}_{\mathrm{i}}=A^{\prime} \frac{1}{3} \mathscr{P}_{1}\right.$, $\left.\mathscr{P}_{2}^{\prime}=D_{1}^{\prime} \mathscr{P}_{2}\right)$. Therefore we have established that if a domain of a Lagrangian manifold associated with a Hamiltonian of the form (1) has a smooth and smoothly invertible projection onto a domain surrounding the origin of momentum space, then that manifold has locally the structure associated with the hyperbolic umbilic catastrophe.

The bifurcation set associated with this catastrophe was shown in Fig. 5. One slice of the bifurcation set (the plane $\mathscr{M}$, corresponding to $a_{3}=0$ ) contains a corner. No other slice has such a corner however, so from the point of view of catastrophe theory, the corner should be unstable in two dimensions - a general perturbation of the manifold would produce a nonzero value of $a_{3}$, and the corner would break up into a fold and a cusp.

The explanation of the observed stability of the corner is again the fact that what is generic or typical in mathematics is not necessarily typical in physics. As was proved earlier, a Hamiltonian of the form (1) cannot admit generators $S\left(P_{1}, P_{2}\right)$ containing quadratic terms in the momenta: the constant $a_{3}$ must vanish, because the kinetic energy contains no terms that are linear in the momenta. Therefore the plane $\mathscr{M}$, containing the corner, is the only physically relevant slice of the bifurcation set. Within the class of Lagrangian manifolds associated with a Hamiltonian of the form (1), the corner is stable - perturbations to the manifold that do not produce quadratic terms in $\hat{S}$ leave the corner intact.

(We may mention that perturbations to the manifold that involve linear terms, $a_{1} \mathscr{P}_{1}+a_{2} \mathscr{P}_{2}$ have no such effect. In fact, from the equations $\mathscr{Q}_{i}=-\partial S / \partial \mathscr{P}{ }_{i}$, we find that these linear terms only correspond to displacements of the origin of coordinates of $\mathscr{Q}$ space. From our point of view, such displacements are of no interest.)

\section{E. Extension to $\boldsymbol{N}$ degrees of freedom}

Our development through Eq. (11) applies to systems with any number of degrees of freedom, while the rest of the 
analysis, Eqs. (12)-(45), applies only to systems with two degrees of freedom. For $N=3$ or higher, we advance the following propositions.

We already proved that for any $N$, a change of variables puts the Hamiltonian in the form (7), and the HamiltonJacobi equation in the form (9). In the Taylor expansion of $\widehat{S}(\mathbf{P})$, linear and quadratic terms were shown to vanish. Therefore the lowest-degree terms in $\hat{S}(\mathbf{P})$ are cubic:

$$
\hat{S}(\mathbf{P})=\frac{1}{6} \sum_{i j k} S_{i j k} P_{i} P_{j} P_{k} .
$$

Here we adopt the convention that the coefficients $S_{i j k}$ are invariant under all permutations of indices,

$$
S_{i j k}=S_{j k i}=S_{k i j}=S_{k j i}=S_{j i k}=S_{i k j} .
$$

For example, the coefficients $S_{112}, S_{121}$, and $S_{211}$ all multiply $P_{1}^{2} P_{2}$; and we take these coefficients to be equal.

As always, $\widehat{S}(\mathbf{P})$ generates a manifold in phase space through the equations,

$$
-Q_{m}(\mathbf{P})=\frac{\partial \hat{S}}{\partial P_{m}}=\frac{1}{2} \sum_{i j} S_{m i j} P_{i} P_{j} .
$$

This manifold must be consistent with the Hamilton-Jacobi equation (9) and this provides restrictions on the coefficients $S_{k i j}$. When Eq. (48) is put into Eq. (9), a set of linear inhomogeneous equations for the $S_{k i j}$ 's is obtained.

A general orthogonal point transformation of coordinates and momenta would be generated by a matrix $\mathscr{R}=\left[\mathscr{R}_{i j}\right]$ according to the rules

$$
\begin{aligned}
P_{i} & =\sum_{j} \mathscr{R}_{i j} \mathscr{P}_{j}, \\
Q_{i} & =\sum_{j} \mathscr{R}_{i j} \mathscr{Q}_{j}, \\
F_{i} & =\sum_{j} \mathscr{R}_{i j} \mathscr{F}_{j} .
\end{aligned}
$$

In the transformed variables $\{\mathscr{P}, \mathscr{Q}\}$, the form of the Hamilton-Jacobi equation is the same as before

$$
\sum_{m} \frac{1}{2} \mathscr{P}_{m}^{2}-\mathscr{F}_{m} \mathscr{Q}_{m}(\mathscr{P})=0 .
$$

We propose that for any $\hat{S}(\mathbf{P})$ locally satisfying the original Hamilton-Jacobi equation, there exists an orthogonal transformation such that in the new variables the Hamilton-Jacobi equation is separated, and

$$
\begin{aligned}
& \frac{1}{2} \mathscr{P}_{m}^{2}-\mathscr{F}_{m} \mathscr{Q}_{m}\left(\mathscr{P}_{m}\right)=0, \\
& \mathscr{Q}_{m}(\mathscr{P})=\mathscr{Q}_{m}\left(\mathscr{P}_{m}\right)=\mathscr{P}_{m}^{2} / 2 \mathscr{F}_{m},
\end{aligned}
$$

and the generator in transformed variables is

$$
\widehat{S}[\mathbf{P}(\mathscr{P})]=\sum_{m} \mathscr{P}_{m}^{3} / 6 \mathscr{F}_{m} .
$$

It is easy to prove that given a separated local solution (52), the inverse transformation $\mathscr{R}^{-1}$ gives a solution to Eq. (9), $\hat{S}(\mathbf{P})$. We also believe (but have not proved) that every local solution $\widehat{S}(\mathbf{P})$ can be obtained by this means. If this is true, then the caustics must locally have the structure described in the next paragraph.

Singular points of the manifold are given by the equa-
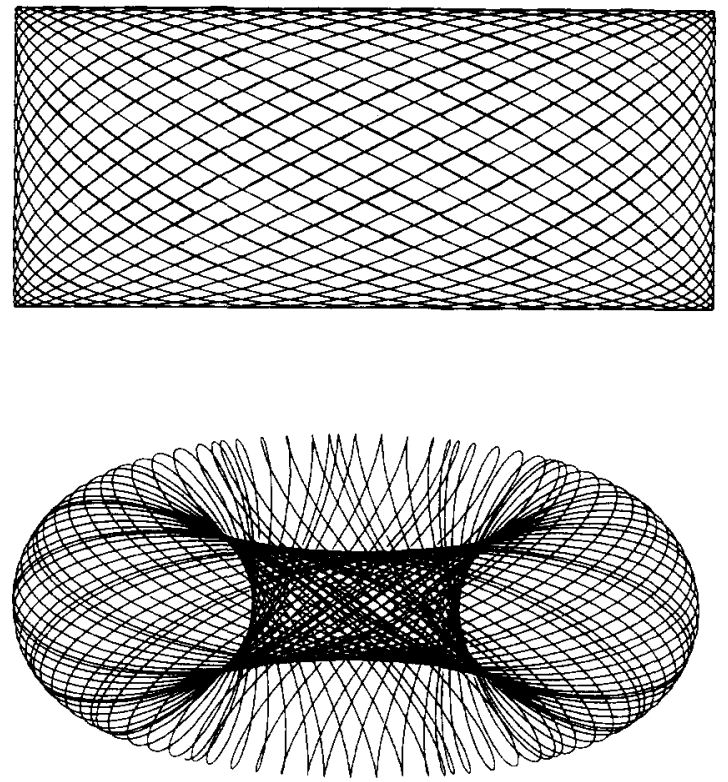

FIG. 10. Trajectories for the Hamiltonian (54) with $k_{1}=1.0, k_{2}=2^{1 / 2}$. Initial conditions for both trajectories were $q_{1}=1.0, q_{2}=1.5, p_{1}=0.0$, $p_{2}=0.0$. Top: $c=0$; bottom: $c=0.523598776$. Each corner breaks up into the typical section of the hyperbolic umbilic.

tion

$$
\frac{\partial(\mathscr{Q})}{\partial(\mathscr{P})}=\prod_{m} \frac{\partial \mathscr{Q}_{m}\left(\mathscr{P}_{m}\right)}{\partial \mathscr{P}_{m}}=0
$$

or

$$
\prod_{m} \mathscr{P}_{m}=0 \text {. }
$$

Hence the projection of the singular points of the manifold into momentum space are the $N$ planes on which any one of the $\mathscr{P}_{m}$ 's vanishes. Each of these planes has dimension $N-1$. The projection of the singular points into configuration space are the $N$ "quadrants" in which any one of the $\mathscr{Q}$ 's vanishes and each of the other $\mathscr{Q}$ 's has a fixed sign. For example in the case $N=3$, if all $\mathscr{F}$ 's are positive then the caustics are three quarter planes: $\mathscr{Q}_{1}=0$ with $\mathscr{Q}_{2}$ and $\mathscr{Q}_{3}$ nonnegative, $\mathscr{Q}_{2}=0$ with $\mathscr{Q}_{1}$ and $\mathscr{Q}_{3}$ nonnegative, and $\mathscr{Q}_{3}=0$ with $\mathscr{Q}_{1}$ and $\mathscr{Q}_{2}$ nonnegative. Locally these caustics have the form of three walls meeting in a corner, as found in Fig. 6(d).

\section{F. An illustrative calculation}

Our theorem, asserting that Hamiltonians having no linear terms in the momenta admit corners as stable structures, can be illustrated very clearly by a simple calculation. Consider the Hamiltonian

$$
H=\frac{1}{2}\left(p_{1}^{2}+p_{2}^{2}\right)+\frac{1}{2}\left(k_{1} q_{1}^{2}+k_{2} q_{2}^{2}\right)+c q_{1} p_{2} .
$$

If $c=0$, this Hamiltonian has the form (1), so the theorem asserts that corners should be stable under changes in the initial conditions and under changes in the force constants. In fact, every trajectory has a rectangular envelope with four corners. If we change $c$ to a nonzero value, then this Hamiltonian no longer has the form (1); catastrophe theory then tells us that corners should no longer be stable, so we expect 
that for the typical trajectory each corner will break up into the fold-and-cusp representing the typical two-dimensional section of the hyperbolic umbilic. This is shown in Fig. 10.

\section{SEMICLASSICAL WAVE FUNCTIONS NEAR A CORNER}

As is well-known, near any caustic, primitive semiclassical wave functions are singular, and more accurate forms are needed. A motivation for the study of the geometry of caustics and their relationship to elementary catastrophes is the desire to obtain simple standard forms for quantum-mechanical wave functions in such singular regions. The classification theorem tells us that a very limited set of standard forms will be needed to describe most physical situations for $N$ small-one for each of the elementary catastrophes. For $N=2$, we need a formula that describes wave functions near folds, and another describing wave functions near cusps. The first of these is well-known to be the Airy function, and the second is called the Pearcey function. Both have now been extensively studied, and Taylor expansions, asymptotic expansions, tables, and graphs are all available. ${ }^{2}$

From this point of view, the analysis given in this paper may appear at first sight to be a bit of bad news. We have shown that typical Hamiltonians for physical systems admit stable structures other than those enumerated by the classification theorem. It follows that these additional structuresthe corners-also have to be analyzed, and standard forms for wave functions near corners also have to be found. The news has a happy ending, however: the leading term in the standard form describing a corner-wave function is nothing more than a product of Airy functions.

We calculate wave functions near a corner using the general framework developed by Maslov and Fedoriuk. $^{3(c), 10}$ They have shown that in the vicinity of almost any singular region, a good approximation to the wave function can be obtained using Fourier transformation from a momentum-space or mixed-space form

$$
\begin{aligned}
\Psi(\mathbf{q})= & (-2 \pi i \hbar)^{-K / 2} \int \exp \left(i \sum_{a \in \alpha} p_{a} q_{a} / \hbar\right) \\
& \times \widetilde{\Psi}\left(\mathbf{p}_{\alpha} \mathbf{q}_{\beta}\right) d \mathbf{p}_{\alpha} .
\end{aligned}
$$

Such a form is useful whenever the primitive semiclassical form in the mixed $\mathbf{p}_{\alpha} \mathbf{q}_{\beta}$ space provides an acceptable approximation:

$\widetilde{\Psi}\left(\mathbf{p}_{\alpha} \mathbf{q}_{\beta}\right)=C\left|\widetilde{J}\left(\mathbf{p}_{\alpha}, \mathbf{q}_{\beta}\right)\right|^{-1 / 2} \exp \left[\tilde{i}\left(\mathbf{p}_{\alpha} \mathbf{q}_{\beta}\right) / \hbar\right]$.

Here $\left\{\mathbf{p}_{\alpha} \mathbf{q}_{\beta}\right\}$ is a mixed set of $N$ coordinates and momenta, with the set containing no canonically conjugate pairs, $\widetilde{S}$ is a generator for the manifold using $\mathbf{p}_{\alpha}$ and $\mathbf{q}_{\beta}$ as fundamental coordinates, $\widetilde{J}$ is a Jacobian corresponding to the inverse of the classical density in the mixed space, and $K$ is the number of variables in the set $\alpha$ (the number of momenta in the chosen mixed space). $C$ is a constant chosen such that this local approximation to $\Psi$ joins smoothly onto local approximations defined in other regions. We refer the reader to Ref. 10 for a detailed discussion, and for calculations based upon this formulation.

Near the corner, since the Lagrange manifold has a good projection into momentum space, $\mathrm{Eq}$. (55) becomes

$$
\widetilde{\Psi}\left(P_{1} P_{2}\right)=C\left|\hat{J}\left(P_{1}, P_{2}\right)\right|^{-1 / 2} \exp \left[\hat{i}\left(P_{1}, P_{2}\right) / \hbar\right],
$$

where now $\widehat{S}$ has the properties discussed in Sec. III.

\section{A. Leading term near the corner}

We have seen that a rotation of coordinates greatly simplifies the generator, so it is best to evaluate $\Psi$ in the rotated coordinates $\mathscr{Q}_{1} \mathscr{Q}_{2}$. Combining Eq. (56) with Eq. (54), we have in these coordinates

$$
\begin{aligned}
\Psi(\mathscr{Q})= & (-2 \pi i \hbar)^{-1} C \int|\mathscr{J}(\mathscr{P})|^{-1 / 2} \\
& \left.\times \exp \{i / \hbar)\left[\mathscr{P}(\mathscr{P})+\mathscr{P}_{1} \mathscr{Q}_{1}+\mathscr{P}_{2} \mathscr{Q}_{2}\right]\right\} d \mathscr{P} \\
= & (-2 \pi i \hbar)^{-1} C \int|\mathscr{J}(\mathscr{P})|^{-1 / 2} \\
& \times \exp \left\{( i / \hbar ) \left[\mathscr{P}_{1} \mathscr{Q}_{1}+\mathscr{P}_{2} \mathscr{Q}_{2}\right.\right. \\
& \left.\left.+\frac{1}{3} A^{\prime} \mathscr{P}_{1}^{3}+\frac{1}{3} D^{\prime} \mathscr{P}_{2}^{3}\right]\right\} d \mathscr{P} .
\end{aligned}
$$

Now if $|\mathscr{J}(\mathscr{P})|^{-1 / 2}$ is approximately constant near the origin $\left(\mathscr{P}_{1}=\mathscr{P}_{2}=0\right)$, it can be taken outside the integral, and then the double integral separates into a product,

$$
\begin{aligned}
\Psi(\mathscr{Q})= & (-2 \pi i \hbar)^{-1} C|\mathscr{J}(0)|^{-1 / 2} \\
& \times \int \exp \left[(i / \hbar)\left(\mathscr{P}_{1} \mathscr{Q}_{1}+\frac{1}{3} A^{\prime} \mathscr{P}_{1}^{3}\right)\right] d \mathscr{P}_{1} \\
& \times \int \exp \left[(i / \hbar)\left(\mathscr{P}_{2} \mathscr{Q}_{2}+\frac{1}{3} D^{\prime} \mathscr{P}_{2}^{3}\right)\right] d \mathscr{P}_{2} \\
= & C^{\prime} \operatorname{Ai}\left(\mathscr{Q}_{1} / A^{\prime 1 / 3} \hbar^{2 / 3}\right) \operatorname{Ai}\left(\mathscr{Q}_{2} / D^{\prime 1 / 3} \hbar^{2 / 3}\right)
\end{aligned}
$$

with

$$
C^{\prime}=i C /\left(\hbar A^{\prime} D^{\prime}\right)^{1 / 3}|\mathscr{J}(0)|^{1 / 2} .
$$

Equation (59) is the promised simple-product-of-Airy functions representing the leading term in the semiclassical approximation to the wave function near a corner. This formula has already been used to calculate a local approximation to a bound state wave function..$^{9(c)}$ It should be obvious that such simple-product forms hold for any number of degrees of freedom, $N$-from Eq. (52) for $\mathscr{S}(\mathscr{P})$, the approximation $\mathscr{J}(\mathscr{P}) \simeq$ constant leads directly to a separated form.

\section{B. Higher-order approximations}

In some cases a more accurate approximation may be desired. The semiclassical approximation can be regarded as an expansion "in powers of $\hbar$," with the primitive semiclassical approximation of order $\hbar^{0}$ and neglected terms of order $\hbar$ and higher. In this view, Eq. (59) is of order $\hbar^{-1 / 3}$, and higher corrections to Eq. (59) lead to an expansion in powers of $\hbar^{1 / 3}$, with the first four terms of order $\hbar^{-1 / 3}, \hbar^{0}, \hbar^{1 / 3}$, and $\hbar^{2 / 3}$. In the present case, these four terms lead to what is called the "uniform approximation." Formulas for this type of uniform approximation have been given in the simpler case of a fold by Chester, Friedman, and Ursel1 ${ }^{11(a)}$ and by Conner, ${ }^{11(b)}$ and formulas for the more complicated case of the full hyperbolic umbilic catastrophe have been given by 
Uzer et al. ${ }^{11}$ Here we give a uniform approximation suitable for a two-dimensional corner. (A closely related formula has been developed and used by Marcus and his collaborators. ${ }^{11(c)}$ Our formula is simpler because we take advantage of a symmetry applicable to bound states but not scattering.)

We choose to work in the variables $Q_{1} Q_{2} P_{1} P_{2}$, with again the corner at the point $(0,0)$.

This formulation of the uniform approximation is based on the assumption that the primitive semiclassical approximation appropriate to the corner is already available. This primitive approximation is a combination of four terms,

$$
\begin{aligned}
\Psi_{p}(\mathbf{Q})= & \sum_{n=1}^{4}\left|J^{(n)}(\mathbf{Q})\right|^{-1 / 2} \\
& \times \exp \left\{(i / \hbar)\left[S^{(n)}(\mathbf{Q})-\mu^{(n)} \frac{\pi}{2}\right]\right\} .
\end{aligned}
$$

The functions $S^{(n)}(Q)$ are generators of the four domains or sheets of the Lagrange manifold that project to the point $\mathbf{Q}$, and $J^{(n)}$ 's are the density Jacobians associated with these domains. The quantities $\mu^{(n)}$ are Maslov indices, which contain the little additional phase shift associated with tunneling into classically forbidden regions. Each of these quantities is described in detail in Refs. 3(c) and 10.

Calculations show that Lagrange manifolds and generators associated with bound states of Hamiltonians of the form (1) usually have certain symmetry properties, which we formulate as follows. If we write $\mathbf{P}^{(n)}(\mathbf{Q})=\nabla S^{(n)}(\mathbf{Q})$, then these functions represent the four possible values of (vector) momentum associated with a given position $\mathbf{Q}-$ i.e., these functions define the embedding of the Lagrange manifold in phase space. It is found that if $P^{(n)}(Q)$ is a sheet of the manifold, then $-\mathbf{P}^{(n)}(\mathbf{Q})$ defines another sheet of the same manifold. [It is obvious that Hamiltonians of the form (1) permit this symmetry; we have not proved that they require this symmetry.] Therefore the four values of $\mathbf{P}^{(n)}(\mathbf{Q})$ are connected in pairs according to the convention

$$
\begin{aligned}
& \mathbf{P}^{(3)}(\mathbf{Q})=-\mathbf{P}^{(1)}(\mathbf{Q}), \\
& \mathbf{P}^{(4)}(\mathbf{Q})=-\mathbf{P}^{(2)}(\mathbf{Q}) .
\end{aligned}
$$

Generators are always defined only to within arbitrary additive constants, and we adopt the convention that the four generators $S^{(n)}(Q)$ have a common value at the corner, ${ }^{12}$ so

$$
\begin{aligned}
& S^{(n)}(\mathbf{Q})=S(0)+\Delta S^{(n)}(\mathbf{Q}), \\
& \Delta S^{(n)}(\mathbf{Q})=\int_{0}^{\mathbf{Q}} \mathbf{P}^{(n)}\left(\mathbf{Q}^{\prime}\right) \cdot d \mathbf{Q}^{\prime},
\end{aligned}
$$

and then the $\Delta S^{(n)}$ 's also have the symmetry (62):

$$
\begin{aligned}
& \Delta S^{(3)}(\mathbf{Q})=-\Delta S^{(1)}(\mathbf{Q}), \\
& \Delta S^{(4)}(\mathbf{Q})=-\Delta S^{(2)}(\mathbf{Q}) .
\end{aligned}
$$

A schematic representation of our conventions is shown in Fig. 9. Following this convention, it can be shown that the Maslov indices are equal to

$$
\begin{aligned}
& \mu^{(1)}=-\mu^{(3)}=1, \\
& \mu^{(2)}=\mu^{(4)}= \pm 2 .
\end{aligned}
$$

The wave function is again represented by an integral,

$$
\begin{aligned}
\Psi(\mathbf{Q})= & \left.(-2 \pi i \hbar)^{-1} \int \hat{J}(\mathbf{P})\right|^{-1 / 2} \\
& \times \exp \{(i / \hbar)[\hat{S}(\mathbf{P})+\mathbf{Q} \cdot \mathbf{P}]\} d \mathbf{P} .
\end{aligned}
$$

Let us now recall that in Eqs. (10) and (12), it was recognized that the Taylor expansion of the generator $S(P)$ contains not only cubic terms, but also all higher terms. These higher terms played no role in the (local) analysis of the structure of the corner, but now they are relevant in the calculation of improved approximations to the wave function-in fact we want to derive a uniform approximation just so that the effects of such higher-order terms in $\hat{S}(\mathbf{P})$ can be incorporated. This time, therefore, we define new variables

$$
\begin{aligned}
& \overline{\mathbf{Q}}=\overline{\mathbf{Q}}(\mathbf{Q}), \\
& \overline{\mathbf{P}}=\overline{\mathbf{P}}(\mathbf{P} ; \mathbf{Q}),
\end{aligned}
$$

and a parameter

$$
A=A(\mathbf{Q})
$$

such that the exponent in Eq. (65) takes exactly the form

$$
\begin{aligned}
& \hat{S}(\mathbf{P})+\mathbf{Q} \cdot \mathbf{P}=\Phi^{0}(\overline{\mathbf{P}} ; \overline{\mathbf{Q}}), \\
& \Phi^{0}(\overline{\mathbf{P}} ; \overline{\mathbf{Q}})=\frac{1}{3} \bar{P}_{1}^{3}+\frac{1}{3} \bar{P}_{2}^{3}+\bar{Q}_{1} \bar{P}_{1}+\bar{Q}_{2} \bar{P}_{2} .
\end{aligned}
$$

[Previously we only put the cubic terms in $\hat{S}(\mathbf{P})$ into a standard form; now we are locally transforming the entire exponent into a standard form.]

This change of variables (66) only makes sense if the Jacobian $\partial\left(P_{1}, P_{2}\right) / \partial\left(\bar{P}_{1}, \bar{P}_{2}\right)$ is finite and nonzero. A necessary condition for this is obtained by differentiating $\mathrm{Eq}$. (67),

$$
\frac{\partial \hat{S}}{\partial P_{i}}+Q_{i}=\sum_{j} \frac{\partial \Phi^{0}}{\partial \bar{P}_{j}} \frac{\partial \bar{P}_{j}}{\partial P_{i}} \quad i=1,2 .
$$

At each $\mathbf{Q}$, there are four values of $\mathbf{P}$ such that the left-hand sides of these equations vanish exactly. Those are the manifold points $\mathbf{P}^{(n)}(\mathbf{Q})$, where $Q_{i}=-\partial \widehat{S} / \partial P_{i}$. At those points, we may regard Eq. (68) as a pair of homogeneous linear equations for unknowns $\partial \Phi^{0} / \partial \bar{P}_{i}$. The determinant of that system of equations is seen to be the Jacobian of the $P_{1} P_{2}$ $\rightarrow \bar{P}_{1} \bar{P}_{2}$ transformation, and since we insist that that determinant must be finite, we are forced to take $\partial \Phi^{0} / \partial \bar{P}_{i}=0$ at these points. Hence from Eq. (67),

$$
\bar{P}_{i}^{2}\left[\mathbf{P}^{(n)}(\mathbf{Q}) ; \mathbf{Q}\right]+\bar{Q}_{i}=0
$$

and

$$
\begin{aligned}
& \hat{S}\left[\mathbf{P}^{(n)}(\mathbf{Q})\right]+\mathbf{P}^{(n)}(\mathbf{Q}) \cdot \mathbf{Q} \\
& \quad=A(\mathbf{Q}) \pm \frac{2}{3} \bar{Q}_{1}^{3 / 2} \pm \frac{2}{3} \bar{Q}_{2}^{3 / 2} .
\end{aligned}
$$

As stated above, for each $\mathbf{Q}$ there are four distinct manifold points $P^{(n)}(Q)$ on which $\mathrm{Eq}$. (70) must hold. Therefore we regard $\mathrm{Eq}(70)$ as a set of four equations determining three unknowns, $\bar{Q}_{1}, \bar{Q}_{2}$, and $A$ at each $\mathbf{Q}$. The existence of a solution to these equations is guaranteed by the symmetry properties (62) and (64); this we prove by constructing a solution. Generators $S(Q)$ and $\widehat{S}(\mathbf{P})$ associated with various representations are related by Legendre transformation, so the left-hand side of Eq. (70) is just

$$
\hat{S}\left[\mathbf{P}^{(n)}(\mathbf{Q})\right]+P^{(n)}(\mathbf{Q}) \cdot \mathbf{Q}=S^{(n)}(\mathbf{Q}) .
$$


Let us take

$$
\begin{aligned}
A(\mathbf{Q})=\frac{1}{4} \sum_{n=1}^{4} S^{(n)}(\mathbf{Q})=S(0), & \\
\frac{2}{3}\left[\bar{Q}_{1}(\mathbf{Q})\right]^{3 / 2} & =\frac{1}{2}\left[S^{(1)}(\mathbf{Q})-S^{(4)}(\mathbf{Q})\right] \\
& =\frac{1}{2}\left[S^{(2)}(\mathbf{Q})-S^{(3)}(\mathbf{Q})\right], \\
\frac{2}{3}\left[\bar{Q}_{2}(\mathbf{Q})\right]^{3 / 2} & =\frac{1}{2}\left[S^{(1)}(\mathbf{Q})-S^{(2)}(\mathbf{Q})\right] \\
& =\frac{1}{2}\left[S^{(4)}(\mathbf{Q})-S^{(3)}(\mathbf{Q})\right]
\end{aligned}
$$

together with the convention that the $\bar{Q}$ 's are negative in the classically allowed region. These $\bar{Q}$ 's obviously satisfy Eq. (7).

As a function of the new variables $Q$, the wave function is now

$\Psi[\mathbf{Q}(\overline{\mathbf{Q}})]=\int \exp \left[i \Phi^{0}(\overline{\mathbf{P}} ; \overline{\mathbf{Q}}) / \hbar\right] g(\overline{\mathbf{P}}) d \overline{\mathbf{P}}$

with

$g(\overline{\mathbf{P}})=(-2 \pi i \hbar)^{-1}|\widehat{J}[\mathbf{P}(\overline{\mathbf{P}})]|^{-1 / 2} \frac{\partial\left(P_{1} P_{2}\right)}{\partial\left(\bar{P}_{1} \bar{P}_{2}\right)}$

We now need to expand $g(P)$ in a series, retaining four terms

$$
\mathrm{g}(\overline{\mathbf{P}})=g_{00}+g_{10} \bar{P}_{1}+g_{01} \bar{P}_{2}+g_{11} \bar{P}_{1} \bar{P}_{2}+\cdots .
$$

[It may seem that the first neglected terms in Eq. (75) involve $\bar{P}_{1}^{2}$ and $\bar{P}_{1}^{2}$, but following the method of Chester $e t$ al., ${ }^{11(a)}$ it is possible to define the series in such a way that quadratic terms involving $\bar{P}_{1}^{2}$ and $\bar{P}_{2}^{2}$ are canceled, so the first neglected terms in Eq. (75) actually are cubic.]

With this approximation, the wave function (73) becomes

$$
\Psi[\mathbf{Q}(\overline{\mathbf{Q}})]=\sum_{m=0}^{1} \sum_{n=0}^{1} g_{m n} U_{m n}(\overline{\mathbf{Q}}),
$$

where

$$
U_{m n}(\overline{\mathbf{Q}})=\int \bar{P}_{1}^{m} \bar{P}_{2}^{n} \exp \left[i \Phi^{0}(\overline{\mathbf{P}} ; \overline{\mathbf{Q}}) / \hbar\right] d \overline{\mathbf{P}} .
$$

Using $\mathrm{Eq}$. (67), these functions $U_{m n}(\mathbf{Q})$ are easily found to be

$$
\begin{aligned}
& U_{00}=\hbar^{2 / 3}(2 \pi)^{2} \operatorname{Ai}\left(\bar{Q}_{1} / \hbar^{2 / 3}\right) \operatorname{Ai}\left(\bar{Q}_{2} / \hbar^{2 / 3}\right), \\
& U_{01}=(-i \hbar)(2 \pi)^{2} \operatorname{Ai}\left(\bar{Q}_{1} / \hbar^{2 / 3}\right) \mathrm{Ai}^{\prime}\left(\bar{Q}_{2} / \hbar^{2 / 3}\right), \\
& U_{10}=(-i \hbar)(2 \pi)^{2} \mathrm{Ai}^{\prime}\left(\bar{Q}_{1} / \hbar^{2 / 3}\right) \mathrm{Ai}\left(\bar{Q}_{2} / \hbar^{2 / 3}\right), \\
& U_{11}=-\hbar^{4 / 3}(2 \pi)^{2} \operatorname{Ai}^{\prime}\left(\bar{Q}_{1} / \hbar^{2 / 3}\right) \mathrm{Ai}^{\prime}\left(\bar{Q}_{2} / \hbar^{2 / 3}\right),
\end{aligned}
$$

where

$$
\operatorname{Ai}^{\prime}(z)=\frac{d \operatorname{Ai}(z)}{d z} .
$$

The last step is to determine the coefficients $g_{m n}$. One good way to choose them is such that the series (75) and the exact formula (74) for $g(P)$ match each other at the stationary points $\mathbf{P}^{(n)}(\mathbf{Q})$. This criterion implies a set of simultaneous linear equations for the coefficients $g_{m n}$, which can be written in the form

$g\left(\overline{\mathbf{P}}^{(n)}\right)=g_{00}+g_{10} P_{1}^{(n)}+g_{01} P_{2}^{(n)}+g_{11} P_{1}^{(n)} P_{2}^{(n)}$.

It follows from the above formulas that this uniform approximation is not in general separable-it cannot necessarily be put into the form $\Psi_{1}\left(Q_{1}\right) \Psi_{2}\left(Q_{2}\right)$.
To summarize, the uniform approximation is given by Eq. (76), where $U$ 's are defined in Eq. (78), $\bar{Q}$ 's are given as functions of $Q$ 's by Eq. (72), and $g$ 's are obtained by solving the simultaneous linear equations (80).

\section{SUMMARY OF RESULTS}

Given: (a) The Hamiltonian has the form (1); (b) the Hamilton-Jacobi equation in momentum space

$$
H\left(\mathrm{p},-\frac{\partial \widetilde{S}}{\partial \mathrm{p}}\right)-E=0
$$

admits as one its solutions a generator $\widetilde{S}(\mathbf{P})$ which is smooth in a neighborhood of the origin $p=0$.

Then:

\section{A. In general}

(1) $\widetilde{S}(p)$ generates a manifold in phase space through the relationship

$$
q_{i}(\mathbf{p})=\frac{-\partial \widetilde{S}(\mathbf{p})}{\partial p_{i}} .
$$

(2) There exist point transformations to (nonunique) "normal" variables $(\mathbf{P}, \mathbf{Q})$ such that the kinetic energy has the form

$$
T=\frac{1}{2} \sum_{i} P_{i}^{2}+\cdots
$$

and such that the point $\mathbf{P}=0$ corresponds through the manifold to $\mathbf{Q}=0$.

(3) When the generator of the manifold is written in these variables, its Taylor expansion about $\mathbf{P}=0$ contains no linear and no quadratic terms.

\section{B. In the case $\boldsymbol{N}=2$}

(4) A rotation of coordinates converts $\widehat{S}(P)$ to the germ of the hyperbolic umbilic catastrophe.

(5) Singular points on the manifold project into momentum space as two straight lines passing through the origin and meeting at a right angle.

(6) Singular points on the manifold project into configuration space as two straight half-lines meeting in a right angle at the origin.

\section{In the case $N=3$}

(7) It is proposed that a rotation of coordinates converts $S(P)$ to a standard form

$$
\widehat{S}(\mathbf{P})=\frac{1}{3} \alpha \mathscr{P}_{1}^{3}+\frac{1}{3} \beta \mathscr{P}_{2}^{3}+\frac{1}{3} \gamma \mathscr{P}_{3}^{3} .
$$

(8) Singular points of this manifold project into momentum space to three perpendicular planes passing through the origin.

(9) These singular points project into configuration space to three perpendicular quadrants (quarter planes) meeting at the origin.

\section{In general}

(10) The leading term in the semiclassical approximation to the wave function associated with a corner is a simple product of Airy functions. 


\section{E. In the case $N=2$}

(11) A uniform approximation suitable in a corner is given by Eqs. (76)-(81).

\section{CONCLUSIONS}

Thom's list of canonical catastrophes describes all types of caustics that are stable under arbitrary smooth perturbations of the Lagrangian manifold (the surface in phase space corresponding to a regular family of classical trajectories). However, the typical Hamiltonian that arises in physics (quadratic in the momenta) does not allow arbitrary perturbations of the manifold. Hence physically stable structures arise which do not correspond to any of Thom's catastrophes. In the study of bound states of typical Hamiltonian systems, to the canonical list of catastrophes one more standard type must be added, which we call a corner. For systems with two degrees of freedom the corner is a special part of the (normally three dimensional) hyperbolic umbilic catastrophe. The analytic geometry of the corner has been described in this paper, and formulas for a uniform approximation to the wave function have been derived.

For bound states of such Hamiltonian systems with two degrees of freedom, the important caustic structures are not folds and cusps, but folds, corners, and swallowtails.

\section{APPENDIX: TRANSFORMATION TO NORMAL VARIABLES}

Transformation to normal variables is done in two steps: first the origin of $\mathbf{q}$ coordinates is changed, and then combinations of $q$ 's are constructed to convert $T\left(q_{0}\right)$ to the unit matrix.

The analysis is quite similar to that used to calculate normal modes of vibration, but there are several differences. (1) The expansion of $H$ is carried out about an extremal point of the motion, where $V\left(q^{0}\right)=E$, not about the equilibrium point. (2) It is not assumed that the trajectory stays close to the point $\mathbf{q}^{0}$. The expansion is only used to describe local properties of the Lagrange manifold. No global assumptions about the manifold are made, except that it corresponds to a regular trajectory. (3) The resulting normal coordinates are not unique, and an arbitrary rotation of normal coordinates leaves them normal. [In contrast, in the usual normal mode analysis, the expansion is carried out about a minimum of $V(q)$, vibrations about this point are presumed to be small enough that higher-order terms are negligible at all times, and the resulting normal coordinates are unique provided that the normal frequencies are nondegenerate.]

The type- 2 generator

$$
W_{A}\left(\mathbf{p}^{\prime}, \mathbf{q}\right)=\sum_{i} p_{i}^{\prime}\left(q_{i}-q_{i}^{0}\right)
$$

with $q^{0}$ defined as the position at which the momenta vanish

$$
q_{i}^{0}=q_{i}(\mathbf{p}=0)=\left.\frac{-\partial \widetilde{S}}{\partial p_{i}}\right|_{\mathrm{p}=0}
$$

leads to the transformation equations

$$
\begin{aligned}
& p_{i}=\frac{\partial W_{A}}{\partial q_{i}}=p_{i}^{\prime}, \\
& q_{i}^{\prime}=\frac{\partial W_{A}}{\partial p_{i}^{\prime}}=q_{i}-q_{i}^{0},
\end{aligned}
$$

and the Hamiltonian can be reexpressed in these variables as

$$
\begin{aligned}
& H=\sum_{i j} \frac{1}{2} T_{i j}^{\prime}\left(\mathbf{q}^{\prime}\right) P_{i}^{\prime} P_{j}^{\prime}+V^{\prime}\left(\mathbf{q}^{\prime}\right), \\
& T_{i j}^{\prime}\left(\mathbf{q}^{\prime}\right) \equiv T_{i j}\left[\mathbf{q}\left(\mathbf{q}^{\prime}\right)\right], \\
& V^{\prime}\left(\mathbf{q}^{\prime}\right) \equiv V\left[\mathbf{q}\left(\mathbf{q}^{\prime}\right)\right], \\
& V^{\prime}(\mathbf{0})=E .
\end{aligned}
$$

Now defining

$$
T_{i j}^{0}=T_{i j}^{\prime}\left(\mathbf{q}^{\prime}=0\right)=T_{i j}\left(\mathbf{q}=\mathbf{q}^{0}\right)
$$

we may make a second linear canonical transformation, using the generator

$$
W_{B}\left(\mathbf{P}, \mathbf{q}^{\prime}\right)=\sum_{i j} P_{i} W_{i j} q_{j}^{\prime}
$$

which gives

$$
\begin{aligned}
& p_{k}^{\prime}=\frac{\partial W_{B}}{\partial q_{k}}=\sum_{i} P_{i} W_{i k}, \\
& Q_{k}=\frac{\partial W_{B}}{\partial P_{k}}=\sum_{j} W_{k j} q_{j}^{\prime} .
\end{aligned}
$$

The kinetic energy term is then

$$
\begin{aligned}
T & =\frac{1}{2} \sum_{i j} p_{i}^{\prime} T_{i j}^{\prime}\left(q^{\prime}\right) p_{j}^{\prime} \\
& =\frac{1}{2} \sum_{i j k l} P_{k} W_{k i} T_{i j}^{\prime}\left(q^{\prime}\right) \widetilde{W}_{j l} P_{l} .
\end{aligned}
$$

We take coefficients $W_{k i}$ such that

$$
W T^{\prime}(0) \tilde{W}=1 \text {. }
$$

This matrix $W$ could be calculated by the following method. Since $T^{0}$ is a symmetric matrix, there exists an orthogonal transformation $X$ which converts it to a diagonal (and positive-definite) matrix

$$
X T^{\prime}(0) \widetilde{X}=t,
$$

where $\widetilde{X}=X^{-1}$ and $t$ is diagonal. Then the matrix $Y=t_{i i}{ }^{-1 / 2} \delta_{i j}$ converts $t$ to the unit matrix:

$$
Y t \tilde{Y}=1 \text {. }
$$

Finally, any orthogonal matrix $Z$ leaves the unit matrix unchanged,

$$
\mathrm{Z} 1 \widetilde{Z}=1 .
$$

Therefore $W$ can be taken to be the product

$$
W=Z Y X
$$

for then

$$
W T^{\prime}(0) \widetilde{W}=Z Y X T^{\prime}(0) \widetilde{X} \widetilde{Y} \widetilde{Z}=1 .
$$

Clearly $W$ is not unique, since the orthogonal transformation $Z$ is arbitrary. It follows that our "normal coordinates" are also not unique, and from any one set of normal coordi- 
nates an arbitrary orthogonal transformation (e.g., rotation) gives another set of normal coordinates.

Let us now denote

$$
W T^{\prime}\left(\mathbf{q}^{\prime}\right) \widetilde{W}=T^{\prime \prime}\left(\mathbf{q}^{\prime}\right) \text {. }
$$

Next, $T^{\prime \prime}\left(q^{\prime}\right)$ and $V\left(q^{\prime}\right)$ have to be reexpressed as functions of $Q$ 's using the inverse of relationship (A9), and then the Hamiltonian in new variables is

$$
\hat{H}(\mathbf{P}, \mathbf{Q})=\frac{1}{2} \sum_{i j} \widehat{T}_{i j}(\mathbf{Q}) P_{i} P_{j}+\hat{V}(\mathbf{Q})
$$

with

$$
\begin{aligned}
& \hat{T}(\mathbf{Q})=T^{\prime \prime}\left[\mathbf{q}^{\prime}(\mathbf{Q})\right], \\
& \hat{V}(\mathbf{Q})=V^{\prime}\left[\mathbf{q}^{\prime}(\mathbf{Q})\right], \\
& \hat{T}(\mathbf{0})=1, \\
& \hat{V}(0)=E
\end{aligned}
$$

in agreement with Eq. (6) of the main text.

\section{ACKNOWLEDGMENTS}

This research was supported by the National Science Foundation and by the Jeffress Foundation. I thank S. K. Knudson for several helpful conversations, R. Waterland for leading me to the polynomial (24), and L. Menges for executing the drawings. Figures $6(\mathrm{a})-6(\mathrm{c})$ were reprinted with permission from Refs. 5(d), 5(a), and 5(g), respectively.

'M. V. Berry, Adv. Phys. 25, 1 (1976).

${ }^{2} J$. N. L. Connor, Mol. Phys. 31, 33 (1976); 26, 1371 (1973); J. N. L. Connor and D. Farrelly, Chem. Phys. Lett. 81, 306 (1981); J. Chem. Phys. 75, 2831 (1976). J. N. L. Connor and R. Marcus, ibid. 55, 5636
(1971).

${ }^{3}$ (a) R. Thom, Structural Stability and Morphogenesis (Benjamin, New York, 1975); (b) V. I. Arnold, Russ. Math. Surv. 23, 1 (1968); 30, (1975); Funct. Anal. Appl. 6, 222, 254 (1972); (c) V. P. Maslov and M. V. Fedoriuk, Semi-Classical Approximation in Quantum Mechanics (Reidel, Boston, 1981).

${ }^{4}$ T. Poston and I. Stewart, Catastrophe Theory and its Applications (Pitman, San Francisco, 1978); (b) p. 94; (c) p. 95; (d) p. 108; R. Gilmore, Catastrophe Theory for Scientists and Engineers (Wiley, New York, 1981).

5(a) D. W. Noid and R. A. Marcus, J. Chem. Phys. 67, 559 (1977); (b) D. W. Noid, M. L. Koszykowski, and R. A. Marcus, ibid. 71, 2864 (1979); (c) 73, 391 (1980); (d) J. Chem. Educ. 57, 624 (1980); (e) Annu. Rev. Phys. Chem. 32, 267 (1981); (f) D. W. Noid, D. M. Wardlaw, M. L. Koszykowski, and R. A. Marcus, J. Phys. Chem. 87, 2733 (1983); (g) J. T. Muckerman, D. W. Noid, and R. A. Marcus, J. Chem. Phys. 78, 3981 (1983).

${ }^{6}$ In his examination of refraction of light from irregular glass, Berry (Ref. 1) found that such swallowtail structures were unusual. In bound trajectories of Hamiltonian systems, they appear very frequently.

'One's first thought might be that the observed stability of the corners and edges might have something to do with separability of the HamiltonJacobi equation (or of the Schrödinger equation). This turns out to be incorrect. None of the trajectories shown in Fig. 6 correspond to separable systems.

${ }^{8}$ The mathematical form of this statement is that the function $\Phi\left(p_{1} p_{2} ; q_{1} q_{2}\right)$ is three-determinate, a fact that emerges from the analysis later, when we show that $\Phi$ can be expressed as a standard form of the hyperbolic umbilic catastrophe.

${ }^{9}$ This statement presumes that the axes $P_{1}$ and $P_{2}$ are orthogonal; otherwise such statements can have no meaning.

${ }^{10}$ (a) J. B. Delos, Adv. Chem. Phys. 65, 161 (1986); (b) S. K. Knudson, J. B. Delos, and B. Bloom, J. Chem. Phys. 83, 5703 (1985); (c) S. K. Knudson, J. B. Delos, and D. W. Noid, ibid. 84, 6886 (1986).

"(a) C. Chester, B. Friedman, and F. Ursell, Proc. Cambridge Philos. Soc. Math. Phys. Sci. 53, 599 (1957); (b) T. Uzer and M. S. Child, Mol. Phys. 46, 1371 (1982); T. Uzer, J. T. Muckerman, and M. S. Child, ibid. 50, 1215 (1983); (c) R. A. Marcus, J. Chem. Phys. 57, 4903 (1972); H. Kreek, R. Ellis, and R. A. Marcus, ibid. 62, 913 (1975).

${ }^{12}$ A different convention was used in Ref. 10(c); there it was assumed that the Maslov phase shifts $\mu_{k}$ were implicitly incorporated into the $S_{k}(\mathbf{Q})$ 's. 